\title{
Self-similar groups and their geometry
}

\author{
Volodymyr Nekrashevych \\ Department of Mathematics, Texas A\&M University, College Station, TX, USA \\ E-mail address: nekrash@math.tamu.edu
}

\section{Introduction}

This is an overview of results concerning applications of self-similar groups generated by automata to fractal geometry and dynamical systems. Few proofs are given, interested reader can find the rest of the proofs in the monograph [Nek05].

We associate to every contracting self-similar action a topological space $\mathcal{J}_{G}$ called limit space together with a surjective continuous map s: $\mathcal{J}_{G} \longrightarrow$ $\partial_{G}$.

On the other hand if we have an expanding self-covering $f: \mathcal{M}_{1} \longrightarrow \mathcal{M}$ of a topological space by its open subset, then we construct the iterated monodromy group (denoted IMG $(f)$ ) of $f$, which is a contracting selfsimilar group.

These two constructions (dynamical system $\left(\mathcal{J}_{G}, \mathrm{~s}\right)$ from a self-similar group and self-similar group IMG $(f)$ from a dynamical system) are inverse to each other. The action of $f$ on its Julia set is topologically conjugate to the action of $\mathrm{s}$ on the limit space $\mathcal{J}_{\mathrm{IMG}(f)}$ (see Theorem 6.1).

We get in this way on one hand interesting examples of groups from dynamical systems (like the "basilica group" IMG $\left(z^{2}-1\right)$, which is a first example of an amenable group not belonging to the class of the subexponentially amenable groups). On the other hand, iterated monodromy groups are algebraic tools giving full information about combinatorics of self-coverings.

The paper has the following structure. Section "Self-similar actions and automata" provides the basic notions from automata theory and theory

The research was supported by the Swiss National Science Foundation and Alexander von Humboldt Foundation. 
of groups acting on rooted trees. It also gives some classical examples of self-similar groups.

Section "Permutational bimodules" develops algebraic tools which are used in the study of self-similar groups. We define the notion of a permutational bimodule, which gives a convenient algebraic interpretation of automata. A closely related notion is virtual endomorphism, which can be used to construct explicit self-similar actions. We describe at the end of the section self-similar actions of free abelian groups and show how they are related to numeration systems on $\mathbb{Z}^{n}$.

Section 4 defines iterated monodromy groups. We show how to compute them (their standard actions) as groups generated by automata.

Section 5 studies contracting self-similar actions and defines their limit spaces $\partial_{G}$. We also prove some basic properties of the limit spaces, limit $G$-spaces and tiles.

The last section shows connections of the obtained results with other topics of Mathematics. Subsection 6.2 shows that Julia sets of post-critically finite rational functions are limit spaces of their iterated monodromy groups. Next two subsections show a connection between topology of the limit spaces and a notion of bounded automata from [Sid00] and construct an iterative algorithm finding approximations of the limit space of actions by bounded automata. In Subsection 6.5 automata generating iterated monodromy groups of complex polynomials are described. We will see in particular, that iterated monodromy groups of complex polynomials are generated by bounded automata, so that the algorithm of the previous subsection can be used to draw topological approximations of the Julia sets of polynomials. We study in the last subsection the limit spaces of free Abelian groups and fit the theory of self-affine "digit" tilings in the framework of self-similar groups and their limit spaces.

\section{Self-similar actions and automata}

\subsection{Spaces of words}

Let $\mathrm{X}$ be a finite set, called alphabet. We denote by $\mathrm{X}^{*}$ the free monoid generated by $\mathbf{X}$. The elements of $\mathbf{X}^{*}$ are words of the form $x_{1} x_{2} \ldots x_{n}$, including the empty word $\emptyset$. The length of a word $v=x_{1} x_{2} \ldots x_{n}$ is denoted $|v|=n$.

The set $\mathrm{X}^{*}$ has a natural structure of a rooted tree. Namely, the root is the empty word $\emptyset$ and every word $v \in \mathbf{X}^{*}$ is connected with the words of the form $v x, x \in \mathbf{X}$. The set $\mathbf{X}^{n}$ of the words of length $n$ is called the $n$th level of the rooted tree $\mathrm{X}^{*}$.

The automorphism group of the tree $\mathrm{X}^{*}$ is denoted Aut $\mathrm{X}^{*}$. 
We denote by $X^{\omega}$ the set of all infinite sequences (words) of the form $x_{1} x_{2} \ldots, x_{i} \in \mathrm{X}$. The space $\mathrm{X}^{\omega}$ is naturally identified with the boundary of the tree $\mathbf{X}^{*}$, i.e., with the set of all infinite paths starting in the root.

The set $X^{\omega}$ is equipped with the direct product topology. The basis of open sets is the collection of all cylindrical sets

$$
a_{1} a_{2} \ldots a_{n} \mathrm{X}^{\omega}=\left\{x_{1} x_{2} \ldots \in \mathrm{X}^{\omega}: x_{i}=a_{i}, 1 \leq i \leq n\right\}
$$

where $a_{1} a_{2} \ldots a_{n}$ runs through $\mathbf{X}^{*}$. The space $\mathbf{X}^{\omega}$ is totally disconnected and homeomorphic to the Cantor set.

We can introduce in a similar way a topology on the set $X^{\omega} \sqcup X^{*}$ choosing the basis of open sets $\left\{v \mathbf{X}^{*} \cup v \mathbf{X}^{\omega}: v \in \mathbf{X}^{*}\right\}$, where $v \mathbf{X}^{*} \cup v \mathbf{X}^{\omega}$ is the set of all words (finite and infinite) beginning with $v$. The topological space $\mathbf{X}^{\omega} \sqcup \mathbf{X}^{*}$ is compact, the set $X^{\omega}$ is closed in it and the set $X^{*}$ is a dense subset of isolated points.

\subsection{Self-similar actions}

Definition 2.1. A faithful action of a group $G$ on $\mathrm{X}^{*}$ (or on $\mathrm{X}^{\omega}$ ) is said to be self-similar if for every $g \in G$ and $x \in \mathbf{X}$ there exist $h \in G$ and $y \in \mathbf{X}$ such that $g(x w)=y h(w)$ for all $w \in \mathbf{X}^{*}\left(\right.$ resp. $\left.\in \mathbf{X}^{\omega}\right)$.

Every self-similar action on $\mathrm{X}^{*}$ is an action by automorphisms of the rooted tree $\mathrm{X}^{*}$ and hence it induces an action by homeomorphisms of the boundary $X^{\omega}$. It is easy to see that the induced action is also self-similar.

In the other direction, if we have a self-similar action of $G$ on $\mathrm{X}^{\omega}$, then applying Definition $2.1|v|$ times we see that for every $g \in G$ and $v \in \mathrm{X}^{*}$ there exists $u \in \mathrm{X}^{|v|}$ and $h \in G$ such that

$$
g(v u)=u h(u)
$$

for all $u \in \mathbf{X}^{\omega}$. It follows that the map $g: v \mapsto u$ defines a self-similar action of $G$ on $\mathrm{X}^{*}$ and that the union of the original action of $G$ on $\mathrm{X}^{\omega}$ with the obtained action on $X^{*}$ is an action by homeomorphisms on $X^{*} \sqcup X^{\omega}$.

We will denote a self-similar action of a group $G$ on $\mathrm{X}^{*}$ (and the corresponding self-similar action on $\left.\mathrm{X}^{\omega}\right)$ by $(G, \mathrm{X})$. We will also identify in some cases the group $G$ with its image in Aut $\mathrm{X}^{*}$ and speak about self-similar automorphism groups of the rooted tree $\mathrm{X}^{*}$, or just self-similar groups. But it should be noted that self-similar group is always meant together with some action on the rooted tree $\mathbf{X}^{*}$.

\subsection{Automata}

The pair $(h, y)$ in Definition 2.1 is determined uniquely by the pair $(g, x)$, since the action is faithful. The map $(g, x) \mapsto(h, y)$ is naturally interpreted 
as an automaton (transducer) with the set of states $G$ over the alphabet X. The formal definition of automata is as follows.

Definition 2.2. An automaton $(A, X)$ (or just $A$ ) consists of

(1) set of states $A$;

(2) alphabet $\mathrm{X}$;

(3) a map $(\lambda, \pi): A \times X \longrightarrow X \times A$.

The coordinates $\lambda: \mathrm{A} \times \mathrm{X} \longrightarrow \mathrm{X}$ and $\pi: \mathrm{A} \times \mathrm{X} \longrightarrow \mathrm{A}$ are called the output and the transition functions of the automaton, respectively.

An automaton is said to be finite if the set of states is finite.

For example, if $(G, \mathrm{X})$ is a self-similar action, then it can be interpreted as an automaton (called the complete automaton of the action) with the set of states $G$ and alphabet $\mathrm{X}$ such that $(\lambda, \pi)$ is the map $(g, x) \mapsto(y, h)$, where $g, h \in G$ and $x, y \in \mathrm{X}$ are as in Definition 2.1, i.e.,

$$
g(x w)=y h(w)
$$

for all $w \in \mathbf{X}^{*}$.

We will write the last equality formally as

$$
g \cdot x=y \cdot h \text {. }
$$

If we identify $g, h \in G$ with the corresponding transformations of $\mathrm{X}^{\omega}$ and the letters $x, y \in \mathrm{X}$ with the transformations $w \mapsto x w$ and $w \mapsto y w$ (the so called creation operators), then (1) becomes a correct equality of products of transformations.

We introduce the following notation for automata. If $(\lambda, \pi)(q, x)=(y, p)$, then we write

$$
q \cdot x=y \cdot p
$$

and

$$
y=q(x), \quad p=\left.q\right|_{x} .
$$

Equation (2) agrees with the notation (1) that we have introduced for the complete automaton of a self-similar action.

It is convenient to define automata using their Moore diagrams. It is a directed labeled graph with the vertices identified with the states of the automaton. If $(\lambda, \pi)(q, x)=(y, p)$ then we have an arrow starting in $q$, ending in $p$ and labeled by $(x, y)$. See Figure 1 for an example.

For more facts on (groups of) automatic transformations see [Eil74, GNS00, Sid98, Sus98]. 


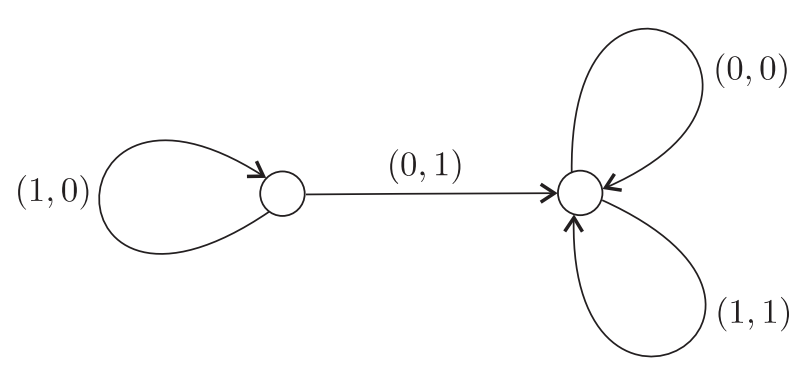

Figure 1. A Moore diagram

\subsection{Automaton $\left(\mathrm{A}, \mathrm{X}^{n}\right)$}

We interpret automata as devices transforming words. If an automaton $(\mathrm{A}, \mathrm{X})$ is in a state $q \in \mathrm{A}$ and it gets on input a finite word $v \in \mathrm{X}^{*}$ then $\mathrm{A}$ reads the first letter $x$ of $v$, gives the letter $q(x)=\lambda(q, x)$ on the output, goes to the state $\left.q\right|_{x}=\pi(q, x)$ and is ready to process the word $v$ further. At the end it will give on the output a word of the same length as $v$ and will stop at some state of $A$.

This procedure can be interpreted as associativity. Namely, if the automaton is in the state $q_{1}$ and gets on the input a word $x_{1} x_{2} \ldots x_{n}$, then we can write

$q_{1} \cdot x_{1} x_{2} \ldots x_{n}=y_{1} \cdot q_{2} \cdot x_{2} \ldots x_{n}=y_{1} y_{2} \cdot q_{3} \cdot x_{3} \ldots x_{n}=\ldots=y_{1} y_{2} \ldots y_{n} \cdot q_{n+1}$, where $q_{i} \cdot x_{i}=y_{i} \cdot q_{i+1}$ for all $1 \leq i \leq n$.

We get at the end an equality

$$
q_{1} \cdot x_{1} x_{2} \ldots x_{n}=y_{1} y_{2} \ldots y_{n} \cdot q_{n+1}
$$

which given us a naturally defined automaton $\left(A, X^{n}\right)$ with the same set of states as the original automaton, but over the alphabet $X^{n}$.

Thus, the output $y_{1} y_{2} \ldots y_{n}=q_{1}\left(x_{1} x_{2} \ldots x_{n}\right)$ and the transition $q_{n+1}=$ $\left.q_{1}\right|_{x_{1} x_{2} \ldots x_{n}}$ of the automaton $\left(\mathrm{A}, \mathrm{X}^{n}\right)$ are defined by the recurrent rules

$$
\begin{array}{rlrl}
\left.q\right|_{\emptyset}=q & & \left.q\right|_{x v}=\left.\left.q\right|_{x}\right|_{v}, \\
q(\emptyset) & =\emptyset & & q(x v)=\left.q(x) q\right|_{x}(v) .
\end{array}
$$

The image $q\left(x_{1} \ldots x_{n}\right)$ of a word $x_{1} \ldots x_{n} \in \mathrm{X}^{*}$ and the state $\left.q\right|_{x_{1} \ldots x_{n}}$ are computed using the Moore diagram of the automaton in the following way. There exists a unique directed path starting in $q$ with the consecutive arrows labeled by $\left(x_{1}, y_{1}\right), \ldots,\left(x_{n}, y_{n}\right)$ for some $y_{1}, \ldots, y_{n} \in \mathbf{X}$. Then $q\left(x_{1} \ldots x_{n}\right)=$ $y_{1} \ldots y_{n}$ and $\left.q\right|_{x_{1} \ldots x_{n}}$ is the end of the path.

The action of $q \in \mathrm{A}$ on the space $\mathrm{X}^{\omega}$ can be defined and computed in a similar way. For every $q$ and $w=x_{1} x_{2} \ldots \in \mathbf{X}^{\omega}$ there exists a unique 
path in the Moore diagram starting at $q$ and labeled by $\left(x_{1}, y_{1}\right),\left(x_{2}, y_{2}\right), \ldots$ for some $y_{1} y_{2} \ldots \in \mathbf{X}^{\omega}$. Then $y_{1} y_{2} \ldots=q\left(x_{1} x_{2} \ldots\right)$. Of course there is no $\left.q\right|_{x_{1} x_{2} \ldots}$.

Note that $q\left(x_{1} x_{2} \ldots\right)$ is the limit of $q\left(x_{1} \ldots x_{n}\right)$ as $n$ goes to infinity, since $y_{1} \ldots y_{n}=q\left(x_{1} \ldots x_{n}\right)$ is a beginning of $q\left(x_{1} x_{2} \ldots\right)$.

Consider, for example, the automaton with the Moore diagram shown on Figure 1. Its right hand side state defines the trivial transformation of the set $\mathbf{X}^{*}$. The left hand side state $a$ acts on the infinite sequence by the rule

$$
a(\underbrace{11 \ldots 1}_{k \text { times }} 0 x_{1} x_{2} \ldots)=\underbrace{00 \ldots 0}_{k \text { times }} 1 x_{1} x_{2} \ldots
$$

This action coincides with the rule of adding 1 to a diadic integer. The transformation $a$ is called the (binary) adding machine or the odometer.

If $(G, \mathrm{X})$ is the complete automaton of a self-similar action, then the action of the automaton $(G, \mathrm{X})$ on the sets $\mathrm{X}^{*}$ and $\mathrm{X}^{\omega}$ coincides with the original action of $G$, just by definition.

In particular, notation $g(v)$ is not ambiguous, it has the same meaning in the sense of the action of $G$ and in the sense of the automaton $\left(G, \mathrm{X}^{n}\right)$. The state $\left.g\right|_{v}$ of the automaton $\left(G, \mathrm{X}^{n}\right)$ is determined by the condition that

$$
g(v w)=\left.g(v) g\right|_{v}(w)
$$

for all $w \in \mathbf{X}^{*}$.

The element $\left.g\right|_{v}$ is called the restriction (or section) of $g$ in $v$. We get the following properties of restrictions as a direct corollary of (5)

$$
\left.g\right|_{v_{1} v_{2}}=\left.\left.\left.g\right|_{v_{1}}\right|_{v_{2}} \quad\left(g_{1} g_{2}\right)\right|_{v}=\left(\left.g_{1}\right|_{g_{2}(v)}\right)\left(\left.g_{2}\right|_{v}\right) .
$$

\subsection{Composition of automata}

If $q_{1}$ is a state of an automaton $\mathrm{A}$ and $q_{2}$ is a state of an automaton $\mathrm{B}$, then the composition of the transformations of $\mathrm{X}^{*}$ defined by the states $q_{i}$ is again a transformation defined by a state of an automaton, called the composition of the automata $\mathrm{A}$ and $\mathrm{B}$.

If $(A, X)$ and $(B, X)$ are two automata over the alphabet $X$, then their composition or product is the automaton, denoted $(\mathrm{A} \cdot \mathrm{B}, \mathrm{X})$, whose set of states is the direct product of $A$ and $B$ and whose transition and output functions are defined by associativity:

$$
q_{1} q_{2} \cdot x=q_{1} \cdot y \cdot p_{2}=z \cdot p_{1} p_{2},
$$

where $q_{2} \cdot x=y \cdot p_{2}$ in $\mathrm{B}$ and $q_{1} \cdot y=z \cdot p_{1}$ in $\mathrm{A}$. 
In other words, the output and transition functions of the automaton A $\cdot B$ are defined by the rules

$$
\begin{gathered}
\left(q_{1} \cdot q_{2}\right)(x)=q_{1}\left(q_{2}(x)\right) \\
\left.\left(q_{1} \cdot q_{2}\right)\right|_{x}=\left.\left.q_{1}\right|_{q_{2}(x)} \cdot q_{2}\right|_{x},
\end{gathered}
$$

where $q_{1} \in \mathrm{A}, q_{2} \in \mathrm{B}$ and the pair $\left(q_{1} \cdot q_{2}\right)$ is hence a state of $\mathrm{A} \cdot \mathrm{B}$.

It is easy to prove by induction that the action of the state $q_{1} \cdot q_{2}$ on $\mathrm{X}^{*}$ is equal to the composition of the actions of $q_{1}$ and $q_{2}$.

An important conclusion is that the set of all transformations of $\mathrm{X}^{*}$ defined by automata is a semigroup under composition. Also the set of transformations of $\mathrm{X}^{*}$ defined by finite automata is a semigroup.

\subsection{Inverse automaton}

Definition 2.3. An automaton $(A, X)$ is said to be invertible if every its state defines an invertible transformation of $X^{*}$.

An automaton is invertible if and only if every its state defines an invertible transformation of $X$. If $(A, X)$ is an invertible automaton, then its inverse is the automaton $\left(A^{-1}, X\right)$, whose set of states is in a bijective correspondence $\mathrm{A}^{-1} \longrightarrow \mathrm{A}: g^{-1} \mapsto g$ with the set of states of $\mathrm{A}$, and

$$
g^{-1} \cdot x=y \cdot h^{-1}
$$

in $\left(A^{-1}, X\right)$ if and only if

$$
g \cdot y=x \cdot h
$$

in $(A, X)$. In particular, if $A$ is finite, then $A^{-1}$ is finite.

If we have the Moore diagram of an invertible automaton $(A, X)$ then the Moore diagram of the inverse automaton $\left(A^{-1}, X\right)$ is obtained by changing every label $(x, y)$ to $(y, x)$. A vertex of the old Moore diagram corresponding to the state $q \in \mathrm{A}$ will correspond to the state $q^{-1} \in \mathrm{A}^{-1}$ in the new diagram.

\subsection{Groups generated by automata}

The following definition gives us a convenient way to construct selfsimilar actions of groups. It was formulated for the first time in the paper [Gri88].

Definition 2.4. Let $(A, X)$ be an invertible automaton. Denote by $\langle A\rangle$ the group generated by the transformations of $X^{*}$ defined by all states of the automaton $\mathrm{A}$. The group $\langle\mathrm{A}\rangle$ is called the group generated by the automaton A.

The group generated by an automaton is always self-similar. 


\subsection{Wreath recursions}

A convenient compact notation for automata and self-similar groups comes from the wreath product decomposition of the automorphism group of the rooted tree $\mathrm{X}^{*}$ into a wreath product of itself with the symmetric group on $\mathrm{X}$.

Unfortunately, the usual usage of this notation is in contradiction with our choice to use left group actions. We switch therefore to right actions $g: v \mapsto v^{g}$ when using wreath recursions.

Definition 2.5. Let $H$ be a group acting (from the right) by permutations on a set $\mathrm{X}$ and let $G$ be an arbitrary group. Then the (permutational) wreath product $G$ wr $H$ is the semi-direct product $G^{\mathrm{X}} \rtimes H$, where $H$ acts on the direct power $G^{\mathrm{X}}$ by the respective permutations of the direct factors.

Every element of the wreath product $G$ wr $H$ can be written in the form $g \cdot h$, where $h \in H$ and $g \in G^{\mathrm{X}}$. If we fix some indexing $\left\{x_{1}, \ldots, x_{d}\right\}$ of the set X, then $g$ can be written as $\left(g_{1}, \ldots, g_{d}\right)$ for $g_{i} \in G$. Here $g_{i}$ is the coordinate of $g$, corresponding to $x_{i}$. Then multiplication rule for elements $\left(g_{1}, \ldots, g_{d}\right) h \in G$ wr $H$ is given by the formula

$$
\left(g_{1}, \ldots, g_{d}\right) \alpha \cdot\left(f_{1}, \ldots, f_{d}\right) \beta=\left(g_{1} f_{1^{\alpha}}, \ldots, g_{d} f_{d^{\alpha}}\right) \alpha \beta,
$$

where $g_{i}, f_{i} \in G, \alpha, \beta \in H$ and $i^{\alpha}$ is the image of $i$ under the action of $\alpha$, i.e., such that $x_{i}^{\alpha}=x_{i^{\alpha}}$.

We have the following well known fact.

Proposition 2.1. Let Aut $\mathrm{X}^{*}$ be the full automorphism group of the rooted tree $\mathbf{X}^{*}$. Fix some indexing $\left\{x_{1}, \ldots, x_{d}\right\}$ of $\mathbf{X}$. Then we have an isomorphism

given by

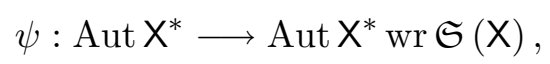

$$
\psi(g)=\left(\left.g\right|_{x_{1}},\left.g\right|_{x_{2}}, \ldots,\left.g\right|_{x_{d}}\right) \alpha
$$

where $\alpha \in \mathfrak{S}(\mathbf{X})$ is the action of $g$ on $\mathbf{X} \subset \mathbf{X}^{*}$.

Here restrictions $\left.g\right|_{x_{i}}$ are defined using (5).

We will usually identify $g$ with its image $\psi(g) \in$ Aut $\mathbf{X}^{*}$ wr $\mathfrak{S}(\mathbf{X})$, so that we write

$$
g=\left(\left.g\right|_{x_{1}},\left.g\right|_{x_{2}}, \ldots,\left.g\right|_{x_{d}}\right) \alpha .
$$

According to this convention, we have Aut $X^{*}=\operatorname{Aut} X^{*}$ wr $\mathfrak{S}(X)$. The subgroup $\left(\text { Aut } X^{*}\right)^{X} \leq$ Aut $X^{*}$ wr $\mathfrak{S}(X)$ is the first level stabilizer $\operatorname{St}(1)$. It acts on the tree $X^{*}$ in the natural way

$$
\left(x_{i} v\right)^{\left(g_{1}, \ldots, g_{d}\right)}=x_{i}\left(v^{g_{i}}\right),
$$

São Paulo J.Math.Sci. 1, 1 (2007), 41-95 
i.e., the $i$ th coordinate of $\left(g_{1}, \ldots, g_{d}\right)$ acts on the $i$ th subtree $x_{i} \mathrm{X}^{*}$.

The subgroup $\mathfrak{S}(X) \leq$ Aut $^{*}$ wr $\mathfrak{S}(X)$ is identified with the group of rooted automorphisms $\alpha=(1, \ldots, 1) \alpha$ acting by the rule

$$
(x v)^{\alpha}=x^{\alpha} v .
$$

Relation (8) is called the wreath recursion. It is a compact way to define finite invertible automata (or, more generally, finitely generated self-similar groups). For example, relation

$$
a=(1, a) \sigma,
$$

where $\sigma$ is the transposition (01) of the alphabet $X=\{0,1\}$, defines an automorphism of the tree $\{0,1\}^{*}$ coinciding with the transformation defined by the state $a$ of the automaton, shown on Figure 1.

In general, every finite invertible automaton with the set of states $\left\{g_{1}, \ldots, g_{n}\right\}$ is described by recurrent formulae:

$$
\left\{\begin{array}{c}
g_{1}=\left(h_{11}, h_{12}, \ldots, h_{1 d}\right) \tau_{1} \\
g_{2}=\left(h_{21}, h_{22}, \ldots, h_{2 d}\right) \tau_{2} \\
\vdots \\
g_{n}=\left(h_{n 1}, h_{n 2}, \ldots, h_{n d}\right) \tau_{n}
\end{array}\right.
$$

where $h_{i j}=\left.g_{i}\right|_{x_{j}}$ and $\tau_{i}$ is the action of $g_{i}$ on X.

\subsection{Examples of self-similar actions}

\subsubsection{Grigorchuk group}

Take $\mathbf{X}=\{0,1\}$. The Grigorchuk group is generated by four automorphisms $a, b, c, d$ of the tree $\mathrm{X}^{*}$, defined recursively by

$$
\begin{array}{ll}
a(0 w)=1 w & a(1 w)=0 w \\
b(0 w)=0 a(w) & b(1 w)=1 c(w) \\
c(0 w)=0 a(w) & c(1 w)=1 d(w) \\
d(0 w)=0 w & d(1 w)=1 b(w)
\end{array}
$$

or in terms of the wreath recursion:

$$
a=\sigma, \quad b=(1, c), \quad c=(1, d), \quad d=(a, b),
$$

where $\sigma \in \mathfrak{S}(\mathbf{X})$ is the transposition.

Hence, the Grigorchuk group is generated by the automaton with the Moore diagram shown on Figure 2.

The Grigorchuk group is an example of an infinite finitely generated torsion group. It is also the first example of a group of intermediate 


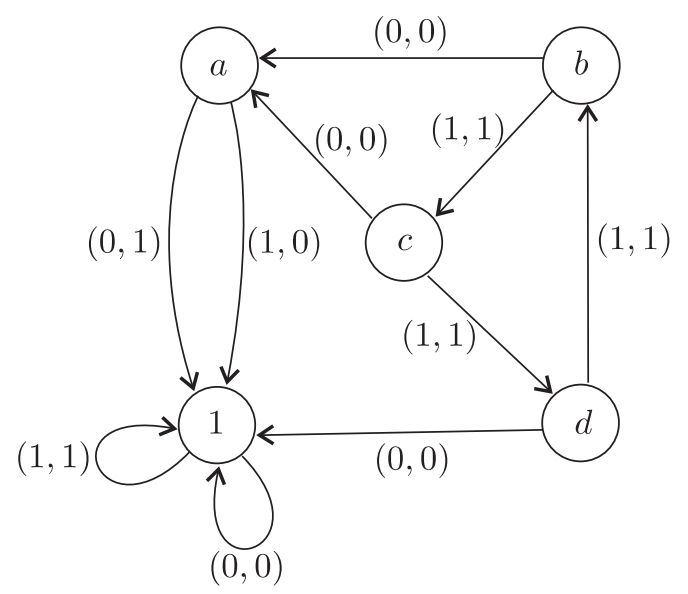

FiguRE 2. The automaton generating the Grigorchuk group

growth (which answers a problem by Milnor). It has many other interesting properties such as just-infiniteness, finite width, etc. See more details in [Gri80, Har00, BGŠO3].

\subsubsection{Gupta-Sidki group}

Let $p$ be an odd prime. The Gupta-Sidki p-group is generated by two automorphisms $a, t$ of the tree $\mathrm{X}^{*}=\{0,1, \ldots, p-1\}^{*}$, defined by the recursion

$$
a=\sigma, \quad t=\left(a, a^{-1}, 1,1, \ldots, 1, t\right),
$$

where $\sigma$ is the cyclic permutation $(0,1, \ldots, p-1) \in \mathfrak{S}(\mathbf{X})$.

It was defined for the first time in [GS83]. The Gupta-Sidki group is also an infinite torsion group. For various properties of this group see the papers [Sid87b, Sid87a, BG00, BG02].

\subsubsection{Lamplighter group}

Consider the group generated by the automaton over the alphabet $\mathbf{X}=$ $\{0,1\}$ shown on Figure 3 .

The following proposition is due to R. Grigorchuk and A. Żuk [GŻo1] (see also a proof in [GNS00]).

Proposition 2.2. The group generated by the automaton shown on Figure 3 is isomorphic to the "lamplighter group", i.e., to the semi-direct product $(\mathbb{Z} / 2 \mathbb{Z})^{\mathbb{Z}} \rtimes \mathbb{Z}$, where $\mathbb{Z}$ acts on $(\mathbb{Z} / 2 \mathbb{Z})^{\mathbb{Z}}$ by the shift, or equivalently, to the wreath product $(\mathbb{Z} / 2 \mathbb{Z})$ wr $\mathbb{Z}$. 


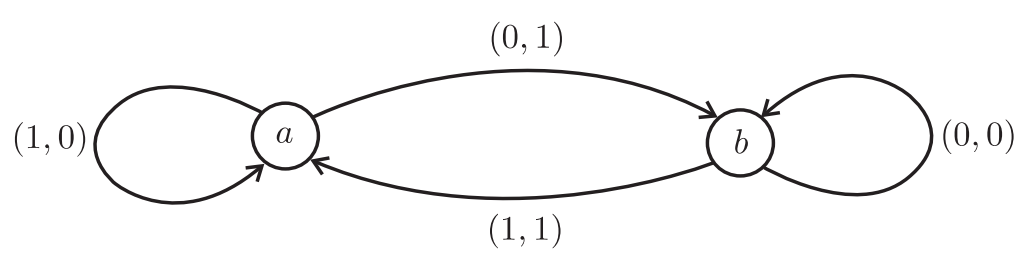

FiguRE 3. The lamplighter group

This action was used in [GZ̈01] to compute the spectrum of the Markov operator on the lamplighter group. It was also used in [GLSŻ00] to construct a counterexample to the strong Atiyah conjecture.

\subsubsection{Free groups}

Consider the automaton shown on Figure 4 over the alphabet $X=\{0,1\}$.

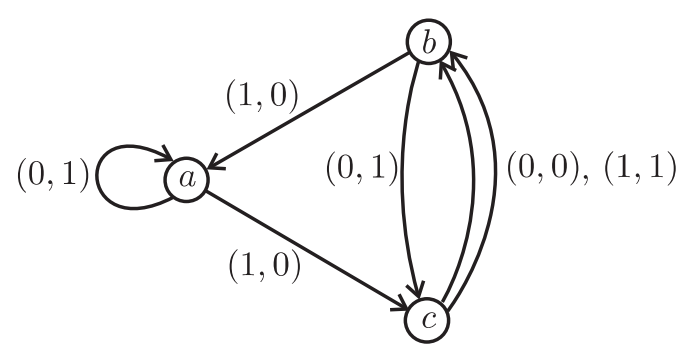

Figure 4

There was posed a conjecture in [Sid00] that the group generated by $a, b, c$ is free. This automaton appeared for the first time in the paper of Aleshin [Ale83]. The conjecture of S. Sidki was proved by Y. Vorobets and M. Vorobets in [VV06].

The first example of a self-similar free group was constructed by Y. Glasner and S. Mozes in [GM05].

The following example was found by Y. Muntyan and D. Savchuk. Consider the automorphisms of the binary tree defined by the wreath recursions

$$
a=(b, b) \sigma, \quad b=(a, c), \quad c=(c, a) .
$$

It is easy to see that the automorphisms $a, b, c$ are involutions. One can actually prove that the group generated by $a, b$ and $c$ is isomorphic to the free product of three groups of order two. Consequently, the group 


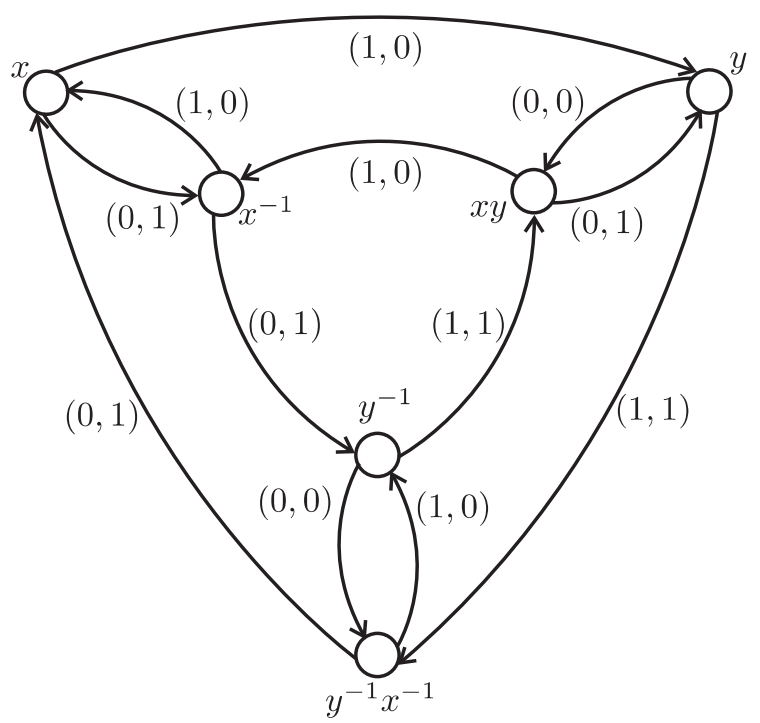

FiguRE 5. Automaton generating $F_{2}$

generated by $x=a b$ and $y=b c$ is free. This free group is self-similar, since

$$
x=\left(x^{-1}, y\right) \sigma, \quad y=\left(x y, y^{-1} x^{-1}\right) .
$$

This free self-similar group is generated by an automaton shown on Figure 5 .

\subsubsection{Multi-dimensional adding machines and linear groups}

Self-similar actions of free Abelian groups $\mathbb{Z}^{n}$ (on a binary tree) where studied in detail in [NS04]. These actions are generalizations of the adding machine action and can be interpreted as numeration systems on $\mathbb{Z}^{n}$. We will describe the construction and this interpretation in Subsection 3.8.

Similar technique is also used to construct self-similar actions of affine groups. The first paper where self-similar actions of affine groups were constructed is [BS98]. Some other examples are given in [NS04].

\section{Permutational bimodules}

\subsection{Definitions}

Definition 3.1. Let $G$ be a group. A permutational $G$-bimodule is a set $\mathfrak{M}$ together with commuting left and right actions of $G$ on $\mathfrak{M}$. Thus we have 
$\operatorname{maps} G \times \mathfrak{M} \longrightarrow \mathfrak{M}:(g, m) \mapsto g \cdot m$ and $\mathfrak{M} \times G \longrightarrow \mathfrak{M}:(m, g) \mapsto m \cdot g$ such that

(1) $1 \cdot m=m \cdot 1=m$ for all $m \in \mathfrak{M}$;

(2) $\left(g_{1} g_{2}\right) \cdot m=g_{1} \cdot\left(g_{2} \cdot m\right)$ and $m \cdot\left(g_{1} g_{2}\right)=\left(m \cdot g_{1}\right) \cdot g_{2}$ for all $g_{1}, g_{2} \in G$ and $m \in \mathfrak{M}$;

(3) $\left(g_{1} \cdot m\right) \cdot g_{2}=g_{1} \cdot\left(m \cdot g_{2}\right)$ for all $g_{1}, g_{2} \in G$ and $m \in \mathfrak{M}$.

Two G-bimodules $\mathfrak{M}_{1}, \mathfrak{M}_{2}$ are isomorphic if there exists a bijection $f$ : $\mathfrak{M}_{1} \longrightarrow \mathfrak{M}_{2}$ which agrees with the left and the right actions, i.e., such that $g \cdot f(m) \cdot h=f(g \cdot m \cdot h)$ for all $g, h \in G$ and $m \in \mathfrak{M}_{1}$.

Let $(G, \mathrm{X})$ be a self-similar action. Then the associated bimodule (or the self-similarity bimodule) is the direct product $\mathfrak{M}=\mathrm{X} \times G$ with the right action given by

$$
(x \cdot g) \cdot h=x \cdot g h,
$$

and the left action

$$
h \cdot(x \cdot g)=\left.h(x) \cdot h\right|_{x} g,
$$

were $x \cdot g$ denotes the element $(x, g)$ of $\mathfrak{M}$. We identify naturally letters $x \in \mathrm{X}$ with the elements $x \cdot 1$ of the bimodule $\mathfrak{M}$.

In other words, we define the bimodule $\mathfrak{M}$ in such a way that the equality $g \cdot x=y \cdot h$ holds in $\mathfrak{M}$ if and only if $g(x w)=y h(w)$ for all $w \in \mathbf{X}^{*}$.

If we identify an element $x \cdot g \in \mathfrak{M}$ with the map

$$
w \mapsto x g(w)
$$

on $\mathrm{X}^{\omega}$, then both left and right actions of $G$ on $\mathfrak{M}$ coincide with composition of maps:

$$
x \cdot g(h(w))=x \cdot g h(w), \quad h(x \cdot g(w))=\left.h(x) \cdot h\right|_{x} g(w) .
$$

Note that the right action of $G$ on the self-similarity bimodule is free (i.e., $m \cdot g=m$ implies $g=1$ ) and has $d=|\mathrm{X}|$ orbits.

We say in general that a $G$-bimodule is a $d$-fold covering bimodule if the right action is free and has $d$ orbits.

Definition 3.2. Self-similar actions $(G, \mathrm{X})$ and $(G, \mathrm{Y})$ are equivalent if their associated $G$-bimodules are isomorphic.

\subsection{Bases of covering bimodules}

Let $\mathfrak{M}$ be a $d$-fold covering $G$-bimodule. A basis of $\mathfrak{M}$ is an orbit transversal of the right action of $G$ on $\mathfrak{M}$, i.e., such a set $\mathrm{X}=\left\{x_{1}, \ldots, x_{d}\right\}$ that every element $m \in \mathfrak{M}$ is written uniquely in the form $x_{i} \cdot g$ for some $x_{i} \in \mathrm{X}$ and $g \in G$. 
If $(G, \mathrm{X})$ is a self-similar action then the alphabet $\mathrm{X}$ is a natural basis of the associated bimodule $\mathfrak{M}=\mathrm{X} \cdot G$. Recall that we identify the letters $x_{i}$ of $\mathrm{X}$ with the elements $x_{i} \cdot 1$ of the bimodule $\mathfrak{M}$.

If $\mathbf{X}=\left\{x_{1}, \ldots, x_{d}\right\}$ is a basis of $\mathfrak{M}$ then a set $\mathbf{Y}=\left\{y_{1}, \ldots, y_{d}\right\}$ is a basis of $\mathfrak{M}$ if and only if there exists a permutation $\pi \in \mathfrak{S}(d)$ and elements $g_{i} \in G$ such that

$$
y_{i}=x_{i^{\pi}} \cdot g_{i}
$$

The left action of $G$ on $\mathfrak{M}$ commutes with the right action, so that we get for every $g \in G$ an automorphism $\psi(g)$ of the right $G$-module $\mathfrak{M}$ :

$$
\psi(g)(m)=g \cdot m \text {. }
$$

The automorphism group of the right $G$-module of $\mathfrak{M}$ is isomorphic to the permutational wreath product $G \operatorname{wr} \mathfrak{S}(\mathbf{X})$, where $\mathbf{X}$ is a basis of $\mathfrak{M}$. Namely, if $\alpha$ is an automorphism of the right module, then the corresponding element of $G$ wr $\mathfrak{S}(\mathrm{X})$ is equal to

$$
\left(g_{1}, g_{2}, \ldots, g_{d}\right) \pi
$$

where $\alpha^{-1}\left(x_{i}\right)=x_{i^{\pi}} \cdot g_{i}$ (we need to take $\alpha^{-1}$, since we pass from a left to right action).

The bimodule $\mathfrak{M}$ is then uniquely determined by the defined structural homomorphism

$$
\psi: G \longrightarrow \text { Aut } \mathfrak{M}_{G} \cong G \mathrm{wr} \mathfrak{S}(\mathrm{X}),
$$

which is called the wreath recursion. On the other hand, every homomorphism

$$
\psi: G \longrightarrow G \text { wr S }(\mathrm{X})
$$

is a structural homomorphism of a $d$-fold covering bimodule.

\subsection{Tensor products of bimodules}

The tensor product $\mathfrak{M}_{1} \otimes \mathfrak{M}_{2}$ of $G$-bimodules $\mathfrak{M}_{1}, \mathfrak{M}_{2}$ is the quotient of the set $\mathfrak{M}_{1} \times \mathfrak{M}_{2}$ by the equivalence relation

$$
\left(x_{1} \cdot g\right) \otimes x_{2}=x_{1} \otimes\left(g \cdot x_{2}\right),
$$

where $g \in G, x_{1} \in \mathfrak{M}_{1}, x_{2} \in \mathfrak{M}_{2}$ and $x \otimes y=(x, y) \in \mathfrak{M}_{1} \times \mathfrak{M}_{2}$.

It is a $G$-bimodule with respect to the actions

$$
g \cdot\left(x_{1} \otimes x_{2}\right)=\left(g \cdot x_{1}\right) \otimes x_{2}, \quad\left(x_{1} \otimes x_{2}\right) \cdot g=x_{1} \otimes\left(x_{2} \cdot g\right) .
$$

Standard arguments show that the tensor product is associative, i.e., that the mapping

$$
\left(x_{1} \otimes x_{2}\right) \otimes x_{3} \mapsto x_{1} \otimes\left(x_{2} \otimes x_{3}\right)
$$

induces an isomorphism $\left(\mathfrak{M}_{1} \otimes \mathfrak{M}_{2}\right) \otimes \mathfrak{M}_{3} \longrightarrow \mathfrak{M}_{1} \otimes\left(\mathfrak{M}_{2} \otimes \mathfrak{M}_{3}\right)$.

The following is straightforward. 
Proposition 3.1. Let $\mathfrak{M}_{1}$ and $\mathfrak{M}_{2}$ be covering bimodules with bases $\mathrm{X}_{1}, \mathrm{X}_{2}$, respectively. Then $\mathfrak{M}_{1} \otimes \mathfrak{M}_{2}$ is a covering bimodule and the set $\mathrm{X}_{1} \otimes \mathrm{X}_{2}=$ $\left\{x_{1} \otimes x_{2}: x_{1} \in \mathbf{X}_{1}, x_{2} \in \mathbf{X}_{2}\right\}$ is its basis.

As a corollary we get that if $X$ is a basis of a bimodule $\mathfrak{M}$, then

$$
\mathrm{X}^{n}=\left\{x_{1} \otimes x_{2} \otimes \cdots \otimes x_{n}: x_{i} \in \mathrm{X}\right\}
$$

is a basis of $\mathfrak{M}^{\otimes n}$. We will use notation

$$
x_{1} x_{2} \ldots x_{n}=x_{1} \otimes x_{2} \otimes \cdots \otimes x_{n} .
$$

Every element of $\mathfrak{M}^{\otimes n}$ is uniquely written in the form $v \cdot g$, where $v \in \mathrm{X}^{n}$ and $g \in G$. In particular, for every pair $g \in G, v \in X^{n}$ there exist a pair $h \in G, u \in \mathrm{X}^{n}$ such that $g \cdot v=u \cdot h$ in $\mathfrak{M}^{\otimes n}$. The pair $u, v$ is uniquely defined due to Proposition 3.1 and we denote $u=g(v)$ and $h=$ $\left.g\right|_{v}$. The following proposition follows directly from the uniqueness and the definitions of permutational bimodules and their tensor products.

Proposition 3.2. The map $v \mapsto g(v)$ described above defines an action of $G$ on the tree $\mathrm{X}^{*}$ by automorphisms. It is the original action of $G$ on $\mathrm{X}^{*}$ if $\mathfrak{M}$ is the bimodule, associated to a self-similar action $(G, \mathrm{X})$. If $g \cdot v=u \cdot h$ in $\mathfrak{M}^{\otimes n}$ then $g(v w)=u h(w)$ for every $w \in \mathbf{X}^{*}$. The restriction map $\left.g \mapsto g\right|_{v}$ satisfies

$$
\left.g\right|_{v_{1} v_{2}}=\left.\left(\left.g\right|_{v_{1}}\right)\right|_{v_{2}},\left.\quad\left(g_{1} g_{2}\right)\right|_{v}=\left.\left.g_{1}\right|_{g_{2}(v)} g_{2}\right|_{v} .
$$

The action of $G$ on $\mathrm{X}^{*}$ is defined by the automaton $(G, \mathrm{X})$ whose output and transition functions are defined by the condition

$$
g \cdot x=\left.g(x) \cdot g\right|_{x} .
$$

The action described in Proposition 3.2 is the self-similar action defined by the bimodule $\mathfrak{M}$ and its basis $\mathrm{X}$. It is denoted $(G, \mathfrak{M}, \mathrm{X})$ or just $(G, \mathrm{X})$.

\subsection{Fock tree of a bimodule}

Let $\mathfrak{M}$ be a permutational G-bimodule. Then its Fock tree (of right orbits) is the set

$$
T_{\mathfrak{M}}=\mathfrak{M}^{*} / G=\bigsqcup_{n \geq 0} \mathfrak{M}^{\otimes n} / G
$$

of right $G$-orbits of the tensor powers of $\mathfrak{M}$. The root of the tree is the unique element of the set $\mathfrak{M}^{\otimes 0} / G=G / G$ and two orbits $A \in \mathfrak{M}^{\otimes n} / G$ and $B \in \mathfrak{M}^{\otimes(n+1)} / G$ are connected by an arrow if there exist $m \in A$ and $x \in \mathfrak{M}$ such that $m \otimes x \in B$.

It is a straightforward corollary of the definition of a tensor product of permutational bimodules that the Fock tree is a well defined rooted tree 
and that the left action of $G$ on the bimodules $\mathfrak{M}^{\otimes n}$ induces an action of $G$ on the Fock tree by automorphisms.

If we fix a basis $\mathrm{X} \subset \mathfrak{M}$, then every vertex of the Fock tree is labeled by a unique word $x_{1} x_{2} \ldots x_{n} \in \mathrm{X}^{*}$ such that $x_{1} \otimes x_{2} \otimes \cdots \otimes x_{n}$ belongs to the corresponding right orbit (the corresponding vertex of the Fock tree). We get hence a bijection between the Fock tree $T_{\mathfrak{M}}$ and the tree $\mathrm{X}^{*}$. It is easy to see that this bijection is an isomorphism of the rooted trees.

The natural (left) action of $G$ on $T_{\mathfrak{M}}$ is conjugated by this isomorphism with the standard action $(G, \mathfrak{M}, \mathbf{X})$.

Recall that two self-similar actions are said to be equivalent, if the associated self-similarity bimodules are isomorphic. The above considerations show that equivalent actions are conjugate.

The following easy proposition gives a recurrent formula for the conjugator.

Proposition 3.3. Let $\mathfrak{M}$ a d-fold covering bimodule over $G$. Let $\mathrm{X}, \mathrm{Y}$ be its bases. Then the self-similar actions $(G, \mathfrak{M}, \mathrm{X})$ and $(G, \mathfrak{M}, \mathrm{Y})$ are conjugate and the conjugating isomorphism is the map $\alpha: \mathrm{X}^{*} \longrightarrow \mathrm{Y}^{*}$ defined by the condition

$$
v=\alpha(v) \cdot \alpha_{v},
$$

where $v \in \mathrm{X}^{*}$ and $\alpha_{v} \in G$. The map $\alpha$ is defined by the recurrent formula

$$
\alpha(x w)=y h_{x} \alpha(w),
$$

where $h_{x} \in G$ and $y \in \mathrm{Y}$ are such that $x=y \cdot h_{x}$ and $w$ is arbitrary.

\subsection{Virtual endomorphisms}

A convenient tool for constructing self-similar actions are virtual endomorphism.

Definition 3.3. A virtual endomorphism $\phi: G \rightarrow G$ of a group $G$ is a homomorphism from a subgroup of finite index $\operatorname{Dom} \phi \leq G$ into $G$. The subgroup Dom $\phi$ is called the domain of the virtual endomorphism. The index $[G: \operatorname{Dom} \phi]$ is called the index of the virtual endomorphism $\phi$ and is denoted Ind $\phi$.

We say that a virtual endomorphism $\phi$ is defined on an element $g \in G$ if $g \in \operatorname{Dom} \phi$. A composition of two virtual endomorphisms $\phi_{1}, \phi_{2}$ is again a virtual endomorphism of index not greater than Ind $\phi_{1} \cdot$ Ind $\phi_{2}$.

Let $(G, \mathrm{X})$ be a self-similar action, which is transitive on the first level $\mathrm{X}^{1}$ of the tree $\mathrm{X}^{*}$. Choose $x \in \mathrm{X}$. Then the associated virtual endomorphism $\phi_{x}$ is defined on the stabilizer of $x \in \mathrm{X}^{*}$ by

$$
\phi_{x}(g)=\left.g\right|_{x} .
$$


It follows that index of $\phi_{x}$ is equal to $d=|\mathrm{X}|$.

For example, the associated virtual endomorphism of the adding machine action is the map $\mathbb{Z} \rightarrow \mathbb{Z}: n \mapsto n / 2$ with the domain equal to the set of even numbers.

In general, if $\mathfrak{M}$ is a $d$-fold covering $G$-bimodule and $x \in \mathfrak{M}$, then the associated virtual endomorphism $\phi_{x}$ is defined by the condition

$$
g \cdot x=x \cdot \phi_{x}(x),
$$

and the domain of $\phi_{x}$ is the subgroup $G_{x}$ of the elements $g \in G$ for which $x$ and $g \cdot x$ belong to the same right orbit.

In the other direction, if $\phi: G \rightarrow G$ is a virtual endomorphism, then it naturally defines an Ind $\phi$-fold covering bimodule denoted $\phi(G) G$. It is the set of formal expressions $\phi\left(g_{1}\right) g_{2}$, for $g_{1}, g_{2} \in G$, where two expressions $\phi\left(g_{1}\right) g_{2}, \phi\left(h_{1}\right) h_{2}$ are considered to be equal if and only if $g_{1}^{-1} h_{1} \in \operatorname{Dom} \phi$ and

in $G$.

$$
\phi\left(g_{1}^{-1} h_{1}\right)=g_{2} h_{2}^{-1}
$$

The bimodule structure on $\phi(G) G$ is given by

$$
\left(\phi\left(g_{1}\right) g_{2}\right) \cdot g=\phi\left(g_{1}\right) g_{2} g
$$

and

$$
g \cdot\left(\phi\left(g_{1}\right) g_{2}\right)=\phi\left(g g_{1}\right) g_{2} .
$$

We can interpret an element $\phi\left(g_{1}\right) g_{2}$ of $\phi(G) G$ as a partially defined transformation

$$
g \mapsto \phi\left(g g_{1}\right) g_{2}
$$

of $G$. Then the left and the right actions of $G$ on $\phi(G) G$ become compositions of these transformations with the right action of $G$ on itself.

If we denote by $x_{0}$ the element $\phi(1) 1$ of $\phi(G) G$, then $g \cdot x_{0}=\phi(g) 1$ belongs to the right orbit of $G$ if and only if $\phi(g) 1=\phi(1) h=x_{0} \cdot h$ for some $h \in G$. But this is equivalent to the condition $g \in \operatorname{Dom} \phi$ and $h=\phi(g)$. Consequently, $\phi$ is the virtual endomorphism associated to the bimodule $\phi(G) G$.

We say that the virtual endomorphisms $\phi_{1}, \phi_{2}: G \rightarrow G$ are conjugate if there exist $g_{1}, g_{2} \in G$ such that Dom $\phi_{1}=g_{1}^{-1}$. Dom $\phi_{2} \cdot g_{1}$ and

$$
\phi_{2}(x)=g_{2}^{-1} \phi_{1}\left(g_{1}^{-1} x g_{1}\right) g_{2}
$$

for all $x \in \operatorname{Dom} \phi_{2}$.

A permutational bimodule $\mathfrak{M}$ is said to be irreducible if for any two $x_{1}, x_{2} \in \mathfrak{M}$ there exist $g_{1}, g_{2} \in G$ such that $g_{1} \cdot x_{1} \cdot g_{2}=x_{2}$.

The following proposition follows directly from the definitions. 
Proposition 3.4. If a virtual endomorphism $\phi$ is associated to an irreducible $G$-bimodule $\mathfrak{M}$, then the bimodules $\phi(G) G$ and $\mathfrak{M}$ are isomorphic.

Virtual endomorphisms $\phi_{1}, \phi_{2}$ of $G$ are conjugate if and only if the bimodules $\phi_{1}(G) G$ and $\phi_{2}(G) G$ are isomorphic.

In particular, any two virtual endomorphisms associated to one bimodule are conjugate.

\subsection{Self-similar actions from virtual endomorphisms}

Let $(G, \mathrm{X})$ be a self-similar action, which is transitive on the first level of the tree $\mathrm{X}^{*}$.

Proposition 3.4 implies that the self-similarity bimodule $\mathfrak{M}=\mathrm{X} \cdot G$ of $(G, \mathrm{X})$ is determined uniquely, up to an isomorphism, by the associated virtual endomorphism $\phi=\phi_{x}$. In other terms, the self-similar action is determined, up to an equivalence (and hence up to a conjugacy), by the virtual endomorphism $\phi$.

Moreover, two self-similar actions $\left(G, \mathrm{X}_{1}\right)$ and $\left(G, \mathrm{X}_{2}\right)$ are equivalent if and only if their associated virtual endomorphisms are conjugate.

We also know that the self-similarity bimodule $\mathrm{X} \cdot G$ is isomorphic to the bimodule $\phi(G) G$.

Let us describe the isomorphism explicitly and show how the self-similar action is computed using the virtual endomorphism.

It is easy to see that a set $\left\{\phi\left(g_{i}\right) h_{i}\right\}_{i=1, \ldots, d}$ is a basis of the bimodule $\phi(G) G$ if and only if the set $\left\{g_{i}\right\}_{i=1, \ldots, d}$ is a left coset transversal of $\operatorname{Dom} \phi$, i.e., if $G$ is the disjoint union of the cosets $g_{i} \operatorname{Dom} \phi$. The sequence $\left\{h_{i}\right\}_{i=1, \ldots, d}$ may be arbitrary.

Proposition 3.5. If $\mathbf{X}=\left\{x_{i}=\phi\left(g_{i}\right) h_{i}\right\}_{i=1, \ldots, d}$ is a basis of the bimodule $\phi(G) G$ then the associated self-similar action $(G, \phi(G) G, \mathrm{X})$ is defined by the formula:

$$
g \cdot x_{i}=x_{j} \cdot h_{j}^{-1} \phi\left(g_{j}^{-1} g g_{i}\right) h_{i},
$$

where $j$ is such that $g_{j}^{-1} g g_{i} \in \operatorname{Dom} \phi$ (i.e., $g g_{i} \in g_{j} \operatorname{Dom} \phi$ ).

If we start from a given self-similar action $(G, \mathrm{X})$, then it may be convenient to know how one gets the elements $g_{i}, h_{i}$ such that $\left\{x_{i}=\phi\left(g_{i}\right) h_{i}\right\}=\mathrm{X}$.

If $\phi$ is associated with $x_{0} \in \mathrm{X}$ (i.e., defined by the condition $g \cdot x_{0}=$ $x_{0} \cdot \phi(g)$ ), then $g_{i}$ and $h_{i}$ have to be chosen so that

$$
g_{i} \cdot x_{0}=x_{i} \cdot h_{i}^{-1},
$$

since the map

$$
\phi\left(g_{i}\right) h_{i} \mapsto g_{i} \cdot x_{0} \cdot h_{i}
$$


is an isomorphism between $\phi(G) G$ and $\mathrm{X} \cdot G$ (which is easy to check using just the definitions).

Corollary 3.6. Let $(G, \mathrm{X})$ be a self-similar action, where $\mathbf{X}=\left\{x_{0}, x_{1}, \ldots\right.$, $\left.x_{d-1}\right\}$, and suppose that it is transitive on the first level of the tree $\mathbf{X}^{*}$. Let $\phi=\phi_{x_{0}}$ be the associated virtual endomorphism and let $g_{i}, h_{i} \in G, 1 \leq i \leq d$ be such that $g_{i} \cdot x_{0}=x_{i} \cdot h_{i}^{-1}$. Then we have for every $g \in G$

$$
g \cdot x_{i}=x_{j} \cdot h_{j}^{-1} \phi\left(g_{j}^{-1} g g_{i}\right) h_{i},
$$

where $j$ is such that $g_{j}^{-1} g g_{i} \in \operatorname{Dom} \phi$.

\subsection{Kernel of a self-similar action}

If we start from an arbitrary virtual endomorphism $\phi: G \rightarrow G$, then in general the associated self-similar action defined in Proposition 3.5 is not necessary faithful. $H$.

We say that a subgroup $H \leq G$ is $\phi$-invariant if $H \leq \operatorname{Dom} \phi$ and $\phi(H) \leq$

Proposition 3.7. The kernel of a self-similar action of a group $G$ with the associated virtual endomorphism $\phi$ is equal to the subgroup

$$
\mathcal{C}(\phi)=\bigcap_{n \geq 1} \bigcap_{g \in G} g^{-1} \cdot \operatorname{Dom} \phi^{n} \cdot g
$$

and is the maximal one among the normal $\phi$-invariant subgroups.

Proof. It follows from the definition of the associated virtual endomorphism that the subgroup Dom $\phi^{n}$ is the stabilizer of the word $x_{0}^{n} \in \mathrm{X}^{*}$, thus the group $\bigcap_{g \in G} g^{-1} \cdot$ Dom $\phi^{n} \cdot g$ is the stabilizer of all the vertices of the $n$th level of the tree $X^{*}$. Therefore, the subgroup (14) is the kernel of the action.

If $N$ is a $\phi$-invariant subgroup of $G$, then it is contained in Dom $\phi^{n}$ for every $n$. If it is normal, then it is contained in every subgroup $g^{-1} \cdot \operatorname{Dom} \phi^{n}$. $g$, thus it is contained in the subgroup (14).

\subsection{Abelian self-similar groups}

Let us illustrate the developed notions and classify self-similar action of free abelian groups, which are transitive on the first level.

The results of this section where obtained (for the case $|X|=2$ ) jointly with S. Sidki in [NS04]. We use additive notation here.

Let $\phi: \mathbb{Z}^{n} \rightarrow \mathbb{Z}^{n}$ be the virtual endomorphism associated to a selfsimilar action of $\mathbb{Z}^{n}$. The map $\phi:$ Dom $\phi \longrightarrow \mathbb{Z}^{n}$ can be extended in a unique way to a linear map $A: \mathbb{Q}^{n} \longrightarrow \mathbb{Q}^{n}$. 
Let us assume for simplicity that $\phi$ is injective (which is always true for faithful self-similar actions) and that the action is recurrent, i.e., that $\phi$ is onto. Then the map $\phi^{-1}$ is defined on the whole group $\mathbb{Z}^{n}$ and is injective, therefore $A^{-1}$ is a matrix with integral entries.

Every element $\phi(r)+h$ of the bimodule $\phi\left(\mathbb{Z}^{n}\right)+\mathbb{Z}^{n}$ can be written in the form $\phi(r+g)$, where $g=\phi^{-1}(h)$. Note that $g \in \operatorname{Dom} \phi$. Consequently, every basis of the bimodule $\phi\left(\mathbb{Z}^{n}\right)+\mathbb{Z}^{n}$ has the form $\mathbf{X}=\left\{x_{0}=\phi\left(r_{0}\right), x_{1}=\right.$ $\left.\phi\left(r_{1}\right), \ldots, x_{d-1}=\phi\left(r_{d-1}\right)\right\}$, where $\left\{r_{i}\right\}$ is a coset transversal of Dom $\phi$.

Then by (11), equality $g \cdot x_{i}=x_{j} \cdot h$ is equivalent to the conditions $g+r_{i}-r_{j} \in \operatorname{Dom} \phi$ and

$$
h=A\left(g+r_{i}-r_{j}\right) .
$$

We say that $\left\{r_{0}, r_{1}, \ldots, r_{d-1}\right\}$ is a digit system of the corresponding selfsimilar action $\left(\mathbb{Z}^{n}, \mathrm{X}\right)$. Recall that if we start from a given self-similar action then a digit system is defined by the condition $r_{i} \cdot x_{0}=x_{i} \cdot \overrightarrow{0}$, where $\overrightarrow{0}$ is the neutral element of the group $\mathbb{Z}^{n}$.

We have the following criterion (see [NS04, BJ99]).

Proposition 3.8. Let $A$ be a linear operator on $\mathbb{Q}^{n}$. Consider the virtual endomorphism $\phi: v \mapsto A(v)$ of the group $\mathbb{Z}^{n}$.

Then the subgroup $\mathcal{C}(\phi)$ is trivial if and only if the characteristic polynomial of $A$ is not divisible by a monic polynomial with integral coefficients (or, in other words, if and only if no eigenvalue of $A$ is an algebraic integer).

As an example, take the $n \times n$ matrix

$$
A=\left(\begin{array}{ccccc}
0 & 1 & 0 & \ldots & 0 \\
0 & 0 & 1 & \ldots & 0 \\
\vdots & \vdots & \vdots & \ddots & \vdots \\
0 & 0 & \ldots & \ldots & 1 \\
1 / 2 & 0 & \ldots & \ldots & 0
\end{array}\right)
$$

The characteristic polynomial of $A$ is $f(x)=x^{n}-1 / 2$ and therefore it defines a virtual endomorphism $\phi: \mathbb{Z}^{n} \rightarrow-\mathbb{Z}^{n}$, giving a faithful self-similar action of $\mathbb{Z}^{n}$ on the binary tree. Let us choose the coset transversal $R=$ $\left\{r_{0}=\overrightarrow{0}, r_{1}=e_{1}=(1,0, \ldots, 0)\right\}$ and let $\mathrm{X}=\left\{0=\phi(\overrightarrow{0})+\overrightarrow{0}, 1=\phi\left(e_{1}\right)+\overrightarrow{0}\right\}$ be the respective basis of the bimodule $\phi\left(\mathbb{Z}^{n}\right)+\mathbb{Z}^{n}$. Let us compute the corresponding self-similar action. 
Let us denote $e_{1}=(1,0, \ldots, 0), e_{2}=(0,1,0, \ldots, 0), \ldots e_{n}=(0, \ldots, 0,1)$. The only generator which does not belong to Dom $\phi$ is $e_{1}$. Then

$$
e_{1}=\left(i d, e_{n}\right) \sigma,
$$

since $e_{1} \cdot 0=1 \cdot \phi\left(e_{1}+r_{0}-r_{1}\right)=1 \cdot \overrightarrow{0}$ and $e_{1} \cdot 1=0 \cdot \phi\left(e_{1}+r_{1}-r_{0}\right)=0 \cdot e_{m}$ by $(15)$.

The action of $e_{i}$ on $\mathrm{X}^{*}$ for $i \geq 2$ is given by the recursion

$$
e_{i}=\left(e_{i-1}, e_{i-1}\right),
$$

since $e_{i} \cdot 0=0 \cdot \phi\left(e_{i}+r_{0}-r_{0}\right)=0 \cdot e_{i-1}$ and $e_{i} \cdot 1=1 \cdot \phi\left(e_{i}+r_{1}-r_{1}\right)=1 \cdot e_{i-1}$.

Thus the defined action of $\mathbb{Z}^{n}$ on $\mathrm{X}^{*}$ is generated by the automaton, shown on Figure 6. It coincides with the adding machine action if $n=1$.

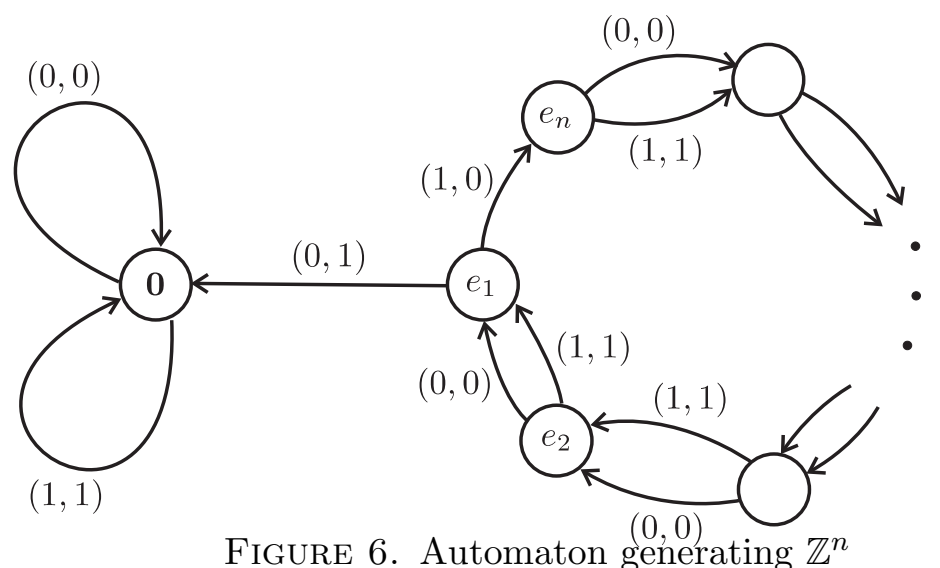

Suppose that $\left(\mathbb{Z}^{n}, \mathrm{X}\right)$ is a self-similar action defined by a virtual endomorphism $\phi: \mathbb{Z}^{n} \rightarrow \mathbb{Z}^{n}$ and a digit system $\left\{r_{0}, \ldots, r_{d-1}\right\}$, where $x_{i} \in \mathrm{X}$ corresponds to $\phi\left(r_{i}\right) \in \phi\left(\mathbb{Z}^{n}\right)+\mathbb{Z}^{n}$. It is natural then to identify a sequence $x_{i_{0}} x_{i_{1}} \ldots \in \mathrm{X}^{\omega}$ with the formal expression

$$
r_{i_{0}}+\phi^{-1}\left(r_{i_{1}}\right)+\phi^{-2}\left(r_{i_{2}}\right)+\cdots+\phi^{-n}\left(r_{i_{n}}\right)+\cdots .
$$

Then the action of $\mathbb{Z}^{n}$ on $X^{\omega}$ will coincide with the formal addition of the elements of $\mathbb{Z}^{n}$ to the expressions of this form. Namely, for every $g \in \mathbb{Z}^{n}$ there exists a unique $r_{j_{0}}$ such that $g+r_{i_{0}} \in \operatorname{Dom} \phi+r_{j_{0}}$ and we can write

$$
g+r_{i_{0}}+\phi^{-1}\left(r_{i_{1}}\right)+\cdots=r_{j_{0}}+\phi^{-1}\left(\phi\left(g+r_{i_{0}}-r_{j_{0}}\right)+r_{i_{1}}\right)+\cdots .
$$


Then there exists a unique $r_{j_{1}}$ such that $g_{1}+r_{i_{1}} \in \operatorname{Dom} \phi+r_{j_{1}}$, where $g_{1}=\phi\left(g+r_{i_{0}}-r_{j_{0}}\right)$, and we can write

$$
g+r_{i_{0}}+\phi^{-1}\left(r_{i_{1}}\right)+\cdots=r_{j_{1}}+\phi^{-1}\left(r_{j_{1}}\right)+\phi^{-2}\left(\phi\left(g_{1}+r_{i_{1}}-r_{j_{1}}\right)\right)+\cdots
$$

and so on. In the limit we get that

$$
g+r_{i_{0}}+\phi^{-1}\left(r_{i_{1}}\right)+\phi^{-2}\left(r_{i_{2}}\right)+\cdots=r_{j_{0}}+\phi^{-1}\left(r_{j_{1}}\right)+\phi^{-2}\left(r_{j_{2}}\right)+\cdots
$$

for some uniquely defined sequence $r_{j_{0}}, r_{j_{1}}, \ldots$ It follows directly from (15) that actually

$$
x_{j_{0}} x_{j_{1}} x_{j_{2}} \ldots=g\left(x_{i_{0}} x_{i_{1}} x_{i_{2}} \ldots\right) .
$$

The formal infinite series that we have used here can be also interpreted as convergent series in the completion of the group $\mathbb{Z}^{n}$ with respect to the sequence of finite index subgroups

$$
\mathbb{Z}^{n}>\operatorname{Dom} \phi>\operatorname{Dom} \phi^{2}>\operatorname{Dom} \phi^{3}>\ldots
$$

\section{Iterated monodromy groups}

\subsection{Definition}

Let $f: \widehat{\mathcal{M}} \longrightarrow \mathcal{M}$ be a covering of an arcwise connected topological space $\mathcal{M}$ by a space $\widehat{\mathcal{M}}$. Consider a basepoint $t \in \mathcal{M}$ and let $\pi_{1}(\mathcal{M}, t)=\pi_{1}(\mathcal{M})$ be the fundamental group. We get then by the classical construction the monodromy action of $\pi_{1}(\mathcal{M})$ on $f^{-1}(t)$. A loop $\gamma \in \pi_{1}(\mathcal{M}, t)$ maps a point $t_{i} \in f^{-1}(t)$ to the end of the unique $f$-preimage of $\gamma$, which starts at $t_{i}$.

It is well known that the monodromy action does not depend, up to a conjugacy, on the choice of the basepoint $t$. In particular, the kernel of the monodromy action does not depend on the basepoint.

Remark. When we multiply two paths $\gamma_{1}$ and $\gamma_{2}$ (for example when $\gamma_{i}$ are elements of the fundamental group) then in the product $\gamma_{1} \gamma_{2}$ the path $\gamma_{2}$ goes in time before $\gamma_{1}$.

A partial self-covering of an arcwise connected and locally arcwise connected topological space $\mathcal{M}$ is a covering $f: \mathcal{M}_{1} \longrightarrow \mathcal{M}$ of $\mathcal{M}$ by its open subset $\mathcal{M}_{1} \subseteq \mathcal{M}$. Then the iterated monodromy group of $f$ is the quotient

$$
\operatorname{IMG}(f)=\pi_{1}(\mathcal{M}) / \bigcap_{n \geq 1} K_{n},
$$

where $K_{n}$ is the kernel of the monodromy action of $\pi_{1}(\mathcal{M})=\pi_{1}(\mathcal{M}, t)$ on the set of preimages of the basepoint $t$ with respect to the $n$th iterate $f^{n}: \mathcal{M}_{n} \longrightarrow \mathcal{M}$ of the covering $f$. 
Profinite (or closed) iterated monodromy group $\overline{\mathrm{IMG}}(f)$ is the completion of the group $\pi_{1}(\mathcal{M})$ with respect to the sequence of subgroups $K_{n}$.

The kernels $K_{n}$ are normal subgroups of finite index. The iterated monodromy group is a dense subgroup of the profinite iterated monodromy group. In particular, iterated monodromy groups are always residually finite.

\subsection{Tree of preimages}

The iterated monodromy group IMG $(f)$ acts naturally on a rooted tree of preimages, constructed in the following way.

Choose a basepoint $t \in \mathcal{M}$. The $n$th level of the tree $T$ is the set $f^{-n}(t)$ of preimages of $t$ under the $n$th iterate $f^{n}$ of $f$. A vertex $z \in f^{-n}(t)$ is connected by an edge with $f(z) \in f^{-(n-1)}(t)$.

If the covering $f: \mathcal{M}_{1} \longrightarrow \mathcal{M}$ is $d$-fold, then every vertex $z \in f^{-(n-1)}(t)$ is adjacent to exactly $d$ vertices of the level $f^{-n}(t)$. These vertices are the $f$-preimages of $z$.

If $\gamma \in \pi_{1}(\mathcal{M}, t)$ is a loop starting and ending in $t$, then for every $n$ and $z \in f^{-n}(t)$ there exists precisely one $f^{n}$-preimage of $\gamma$ starting at $z$. Let us denote it by $\gamma_{z}$ and let $\gamma(z)$ be the end of $\gamma_{z}$. Then the map

$$
z \mapsto \gamma(z)
$$

is an automorphism of the rooted tree $T$. See Figure 7.

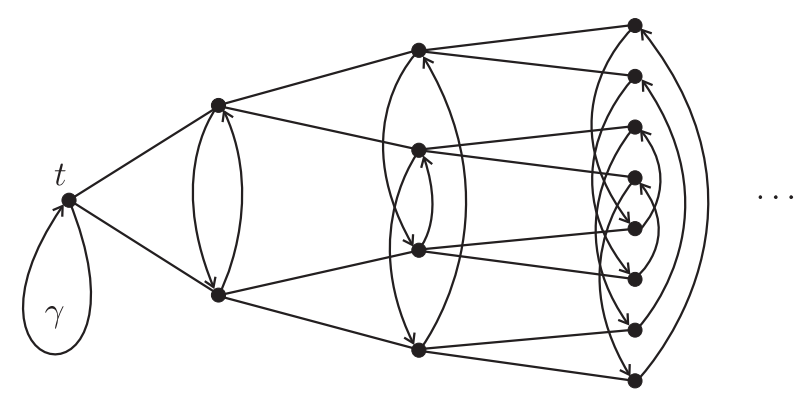

FiguRE 7. Iterated monodromy action

We get in this way an action of the fundamental group $\pi_{1}(\mathcal{M}, t)$ on the tree $T$. This actions is called the iterated monodromy action of $\pi_{1}(\mathcal{M})$. The quotient of the fundamental group by the kernel of the iterated monodromy action is, by definition, the iterated monodromy group IMG $(f)$. 


\subsection{Bimodule of a partial self-covering}

Let $f: \mathcal{M}_{1} \longrightarrow \mathcal{M}$ be a partial self-covering. Choose a basepoint $t \in \mathcal{M}$. Let $\mathfrak{M}(f)$ be the set of the homotopy classes of paths in $\mathcal{M}$ starting in $t$ and ending in a point of $f^{-1}(t)$. Then the set $\mathfrak{M}(f)$ has a structure of a $\pi_{1}(\mathcal{M}, t)$-bimodule. The right action is the natural one:

$$
\ell \cdot \gamma=\ell \gamma .
$$

The path $\ell \gamma$ is a well defined element of $\mathfrak{M}(f)$, since the end of $\gamma$ is a beginning of $\ell$.

The left action is obtained by taking preimages of loops under the covering. Let us denote by $f^{-1}(\gamma)[z]$ the $f$-preimage of a loop $\gamma \in \pi_{1}(\mathcal{M}, t)$ starting at $z \in f^{-1}(t)$. Then we define

$$
\gamma \cdot \ell=f^{-1}(\gamma)[z] \ell .
$$

The right action of $\pi_{1}(\mathcal{M})$ on the bimodule $\mathfrak{M}(f)$ is free and has $d$ orbits (two paths belong to one orbit if and only if they end in a common point). Hence, $\mathfrak{M}(f)$ is a $d$-fold covering bimodule. A collection $\mathbf{X}=\left\{\ell_{1}, \ldots, \ell_{d}\right\}$ is a basis of $\mathfrak{M}(f)$ if and only if the ends of $\ell_{i}$ are pairwise different and hence are all the $f$-preimages of $t$.

Fix some element $\ell \in \mathfrak{M}(p)$ and let $z \in f^{-1}(t)$ be the end of $\ell$. The element $\ell$ defines a virtual endomorphism $\phi$ of $\pi_{1}(\mathcal{M}, t)$ associated with $\mathfrak{M}(f)$. Its domain is the set of loops $\gamma \in \pi_{1}(\mathcal{M})$ such that $f^{-1}(\gamma)[z]$ is also a loop. Thus Dom $\phi$ is an index $d$ subgroup, isomorphic to the fundamental group of $\mathcal{M}_{1}$. The action of the associated virtual endomorphism on its domain is given by

$$
\phi(\gamma)=\ell^{-1} f^{-1}(\gamma)[z] \ell .
$$

We say that $\phi$ is the virtual endomorphism associated with the partial self-covering $f: \mathcal{M}_{1} \longrightarrow \mathcal{M}$ (and the path $\ell$ ).

Proposition 4.1. The virtual endomorphism $\phi$ of $\pi_{1}(\mathcal{M})$ is up to a conjugacy uniquely determined by the partial self-covering $f: \mathcal{M}_{1} \longrightarrow \mathcal{M}$.

The $\pi_{1}(\mathcal{M})$-bimodule $\mathfrak{M}(p)$ is isomorphic to $\phi\left(\pi_{1}(\mathcal{M})\right) \pi_{1}(\mathcal{M})$ and is determined uniquely (up to an isomorphism of bimodules) by the self-covering $f$.

Proof. The virtual endomorphism $\phi$ is the composition of the homomorphisms

$$
\pi_{1}(\mathcal{M}, t) \stackrel{\stackrel{f_{*}^{-1}}{\rightarrow}}{\rightarrow} \pi_{1}\left(\mathcal{M}_{1}, z\right) \stackrel{e_{*}}{\longrightarrow} \pi_{1}(\mathcal{M}, z) \stackrel{L}{\rightarrow} \pi_{1}(\mathcal{M}, t),
$$

where $f_{*}^{-1}$ is the isomorphism $\gamma \mapsto f^{-1}(\gamma)[z]$ of a subgroup of finite index in $\pi_{1}(\mathcal{M})$ with $\pi_{1}\left(\mathcal{M}_{1}\right), e_{*}$ is the homomorphism induced by the embedding 
$\mathcal{M}_{1} \hookrightarrow \mathcal{M}$ and $L$ is the isomorphism of $\pi_{1}(\mathcal{M}, z)$ with $\pi_{1}(\mathcal{M}, t)$ given by the path $\ell$, i.e., the map $\gamma \mapsto \ell^{-1} \gamma \ell$.

It is easy to see that the (virtual) homomorphisms $f_{*}^{-1}, e_{*}$ and $L$ depend, up to inner automorphisms of the fundamental groups, only on the partial self-covering $f$.

The rest follows now from Proposition 3.4.

\subsection{Standard actions}

The tree of preimages $T$ defined by a partial self-covering $f: \mathcal{M}_{1} \longrightarrow \mathcal{M}$ is a $d$-regular rooted tree. Therefore, $T$ is isomorphic to the tree of words $\mathrm{X}^{*}$ over an alphabet $\mathbf{X}$ of $d$ letters. Such an isomorphism is necessary if we want to compute the iterated monodromy action of $\pi_{1}(\mathcal{M})$ on the tree of preimages.

We are going to define a class of nice isomorphisms $\Lambda: \mathrm{X}^{*} \longrightarrow T$ such that the conjugate action of $\pi_{1}(\mathcal{M})$ (and IMG $(f)$ ) on $\mathrm{X}^{*}$ is self-similar.

Proposition 4.2. Let $f_{1}: \mathcal{M}_{1} \longrightarrow \mathcal{M}$ and $f_{2}: \mathcal{M}_{2} \longrightarrow \mathcal{M}$ be partial self-coverings. Then the bimodules $\mathfrak{M}\left(f_{1}\right) \otimes \mathfrak{M}\left(f_{2}\right)$ and $\mathfrak{M}\left(f_{1} \circ f_{2}\right)$ are isomorphic. The isomorphism is the map

$$
L: \ell_{1} \otimes \ell_{2} \mapsto f_{2}^{-1}\left(\ell_{1}\right) \ell_{2},
$$

where $f_{2}^{-1}\left(\ell_{1}\right)$ is the $f_{2}$-preimage of the path $\ell_{1}$ starting at the endpoint of $\ell_{2}$.

Here $\mathfrak{M}\left(f_{1}\right)$ and $\mathfrak{M}\left(f_{2}\right)$ are defined using a common basepoint $t \in \mathcal{M}$.

Proof. We have to show that $L$ is well defined, bijective and agrees with the bimodule structures.

Let us prove that $L$ is well defined. Suppose that $\ell_{1} \otimes \ell_{2}$ and $\ell_{1}^{\prime} \otimes \ell_{2}^{\prime}$ are equal elements of $\mathfrak{M}\left(f_{1}\right) \otimes \mathfrak{M}\left(f_{2}\right)$. This means that there exists an element $\gamma \in \pi_{1}(\mathcal{M}, t)$ such that $\ell_{1}^{\prime}=\ell_{1} \cdot \gamma$ and $\gamma \cdot \ell_{2}^{\prime}=\ell_{2}$. We have $\ell_{1} \cdot \gamma=\ell_{1} \gamma$ and $\gamma \cdot \ell_{2}^{\prime}=f_{2}^{-1}(\gamma)[z] \ell_{2}^{\prime}$, where $z$ is the end of the path $\ell_{2}^{\prime}$. Therefore

$$
\begin{gathered}
L\left(\ell_{1} \otimes \ell_{2}\right)=f_{2}^{-1}\left(\ell_{1}\right) \ell_{2}= \\
f_{2}^{-1}\left(\ell_{1}\right) f_{2}^{-1}(\gamma) \ell_{2}^{\prime}=f_{2}^{-1}\left(\ell_{1} \gamma\right) \ell_{2}^{\prime}=f_{2}\left(\ell_{1}^{\prime}\right) \ell_{2}^{\prime}=L\left(\ell_{1}^{\prime} \otimes \ell_{2}^{\prime}\right),
\end{gathered}
$$

where we choose the $f_{2}$-preimages of the respective paths so that the products are well defined paths in $\mathcal{M}$. See the left-hand side part of Figure 8.

Let us show that $L$ is injective. Suppose that $L\left(\ell_{1} \otimes \ell_{2}\right)=L\left(\ell_{1}^{\prime} \otimes \ell_{2}^{\prime}\right)$. This means that the paths $f_{2}^{-1}\left(\ell_{1}\right)[z] \ell_{2}$ and $f_{2}^{-1}\left(\ell_{1}^{\prime}\right)\left[z^{\prime}\right] \ell_{2}^{\prime}$ are homotopic. Here $z$ and $z^{\prime}$ are ends of the paths $\ell_{2}$ and $\ell_{2}^{\prime}$. In particular the endpoints of 

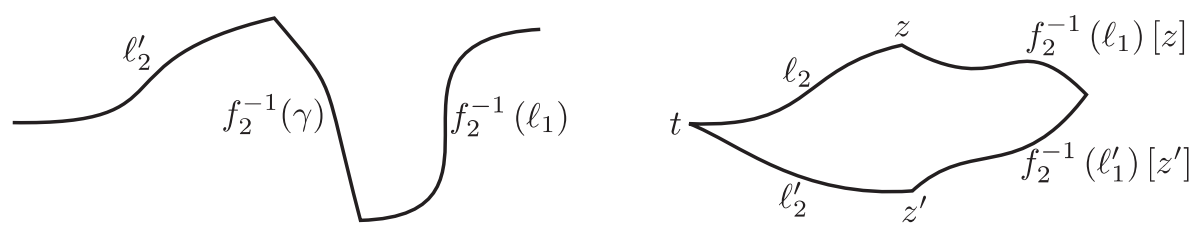

Figure 8

the paths. It follows that $\left(f_{2}^{-1}\left(\ell_{1}^{\prime}\right)\left[z^{\prime}\right]^{-1}\right)^{-1} f_{2}^{-1}\left(\ell_{1}\right)[z]$ is a path homotopic to the path $\ell_{2}^{\prime} \ell_{2}^{-1}$ (see the right-hand side part of Figure 8).

Then

$$
\gamma=f_{2}\left(\left(f_{2}^{-1}\left(\ell_{1}^{\prime}\right)\left[z^{\prime}\right]^{-1}\right)^{-1} f_{2}^{-1}\left(\ell_{1}\right)[z]\right)=\left(\ell_{1}^{\prime}\right)^{-1} \ell_{1}
$$

is a loop such that

$$
\ell_{1}^{\prime} \cdot \gamma=\ell_{1}
$$

and

$$
\gamma \cdot \ell_{2}=f_{2}^{-1}(\gamma)[z]=\ell_{2} \ell_{2}^{-1} \ell_{2}=\ell_{2}^{\prime},
$$

hence $\ell_{1} \otimes \ell_{2}=\ell_{1}^{\prime} \otimes \ell_{2}^{\prime}$.

Let us show that $L$ is surjective. Suppose that $\ell \in \mathfrak{M}\left(f_{1} \otimes f_{2}\right)$ be an arbitrary element, i.e., a path starting at $t$ and ending in some point $t^{\prime} \in$ $\left(f_{1} \otimes f_{2}\right)^{-1}(t)$. Choose some path $\ell_{2} \in \mathfrak{M}\left(f_{2}\right)$ starting at $t$ and ending in some $f_{2}$-preimage of $t$. Then $f_{2}\left(\ell \ell_{2}^{-1}\right)$ is a path starting in $t$ and ending in some $f_{1}$-preimage of $t$. Let us denote it $\ell_{1}$. Then $\ell_{1} \in \mathfrak{M}\left(f_{1}\right)$ and

$$
L\left(\ell_{1} \otimes \ell_{2}\right)=f_{2}^{-1}\left(\ell_{1}\right) \ell_{2}=\ell \ell_{2}^{-1} \ell_{2}=\ell .
$$

It remains only to show that $L$ agrees with the bimodule structures. The equality

is trivial.

$$
L\left(\ell_{1} \otimes \ell_{2} \cdot \gamma\right)=L\left(\ell_{1} \otimes \ell_{2}\right) \cdot \gamma
$$

Let us show that $L$ agrees with the left actions. The path $\gamma \cdot \ell_{1}$ is, by definition the path of the form $f_{1}^{-1}(\gamma) \ell_{1}$. Then the path $L\left(\gamma \cdot \ell_{1} \otimes \ell_{2}\right)$ is the path of the form $f_{2}^{-1}\left(f_{1}^{-1}(\gamma) \ell_{1}\right) \ell_{2}$, where, as usual, we choose the preimages such that the respective products are well defined paths. We have therefore that

$$
L\left(\gamma \cdot \ell_{1} \otimes \ell_{2}\right)=\left(f_{1} \circ f_{2}\right)^{-1}(\gamma) f_{2}^{-1}\left(\ell_{1}\right) \ell_{2}=\gamma \cdot L\left(\ell_{1} \otimes \ell_{2}\right),
$$

that is, $L$ agrees also with the left action. 
Recall that a set of paths $\left\{\ell_{1}, \ldots, \ell_{d}\right\}$ is a basis of $\mathfrak{M}(f)$ if and only if the paths $\ell_{i}$ start in $t$ and end in $t_{i}$, where $\left\{t_{1}, \ldots, t_{d}\right\}=f^{-1}(t)$. So, if $\mathrm{X}=\left\{x_{1}=\ell_{1}, \ldots, x_{d}=\ell_{d}\right\}$ is a basis of the bimodule $\mathfrak{M}(f)$, then $L\left(\mathbf{X}^{n}\right)$ is a basis of $\mathfrak{M}\left(f^{n}\right)$. We have two important conclusions. First is that the map

$$
v \mapsto \text { end of } L(v)
$$

is a bijection $\Lambda: \mathrm{X}^{n} \longrightarrow f^{-n}(t)$. It follows directly from the construction of the isomorphism $L: \mathfrak{M}(f)^{\otimes n} \longrightarrow \mathfrak{M}\left(f^{n}\right)$ that $f\left(\Lambda\left(x_{i_{1}} \ldots x_{i_{n}}\right)\right)=$ $\Lambda\left(x_{i_{1}} \ldots x_{i_{n-1}}\right)$, since the path $L\left(x_{i_{1}} \ldots x_{i_{n-1}} \otimes x_{i_{n}}\right)$ is equal to the path $f^{-1}\left(L\left(x_{i_{1}} \ldots x_{i_{n-1}}\right)\right) \ell_{i_{n}}$. This implies that the map $\Lambda: \mathbf{X}^{*} \longrightarrow T$ is an isomorphism of the rooted trees.

The second conclusion is that the bijection $\Lambda$ conjugates the associated self-similar action $\left(\pi_{1}(\mathcal{M}, t), \mathfrak{M}(f), \mathrm{X}\right)$ with the iterated monodromy action of $\pi_{1}(\mathcal{M}, t)$ on the tree $T=\bigsqcup_{n \geq 0} f^{-n}(t)$, since the left action of $\pi_{1}(\mathcal{M}, t)$ on $\mathfrak{M}^{\otimes n}(f) \cong \mathfrak{M}\left(f^{n}\right)$ coincides with the monodromy action. The action $\left(\pi_{1}(\mathcal{M}, t), \mathfrak{M}(f), \mathrm{X}\right)$ is called the standard self-similar action of $\pi_{1}(\mathcal{M})$ (or of IMG $(f)$, if we quotient it by the kernel of the action).

Computing the standard action is an effective way to compute the iterated monodromy action in terms of automata theory and self-similar groups.

The explicit formula for the standard action defined by a basis follows directly from the definition of the bimodule $\mathfrak{M}(f)$ (see 4.3) and from the definition of self-similar actions associated to bimodules.

Proposition 4.3. Let $\mathrm{X}=\left\{x_{1}=\ell_{1}, \ldots, x_{d}=\ell_{d}\right\}$ be a basis of the bimodule $\mathfrak{M}(f)$, i.e., a collection of paths $\ell_{i}$ starting at $t \in \mathcal{M}$ and ending in its preimages $z_{i} \in f^{-1}(t)$. Then for every $\gamma \in \pi_{1}(\mathcal{M}, t), x_{i} \in \mathbf{X}$ and $v \in \mathbf{X}^{*}$ the following equality holds for the standard action of $\pi_{1}(\mathcal{M})$ (and of $\operatorname{IMG}(f)$ ) on $\mathrm{X}^{*}$ :

$$
\gamma\left(x_{i} v\right)=x_{j}\left(\ell_{j}^{-1} \gamma_{i} \ell_{i}\right)(v)
$$

where $\gamma_{i}=f^{-1}(\gamma)\left[z_{i}\right]$ and $x_{j}=\ell_{j} \in \mathbf{X}$ ends in the same point $z_{j}$ as $\gamma_{i}$.

See Figure 9 where the loop $\ell_{j}^{-1} \gamma_{i} \ell_{i}$ is drawn.

\subsection{An example of computation of IMG $(p)$}

Consider the polynomial $f(z)=z^{2}-1$ as a branched covering of the complex plane $\mathbb{C}$. The only critical point of $z^{2}-1$ is 0 . Its orbit under action of $f(z)$ is $0 \mapsto-1 \mapsto 0$. Hence, $f(z)$ is a covering of the space $\mathcal{M}=\mathbb{C} \backslash\{0,-1\}$ by the open subset $\mathcal{M}_{1}=\mathbb{C} \backslash\{0,-1,1\}$. 


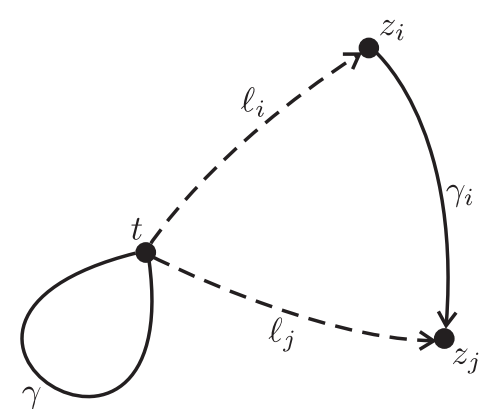

FiguRE 9. Recurrent formula of the standard action

Let us choose a basepoint $t=\frac{1-\sqrt{5}}{2}$. Its $f$-preimages are $t$ and $-t$. Choose $x_{0}=\ell_{0}$ to be the trivial path at $t$ and $x_{1}=\ell_{1}$ to be the path, connecting $t$ with $-t$ above the real axis, as on the lower part of Figure 10 . Let $a$ and $b$ be the generators of $\pi_{1}(\mathcal{M}, t)$ equal to the loops going in the positive direction around the points -1 and 0 , as it is shown on the upper part of Figure 10.

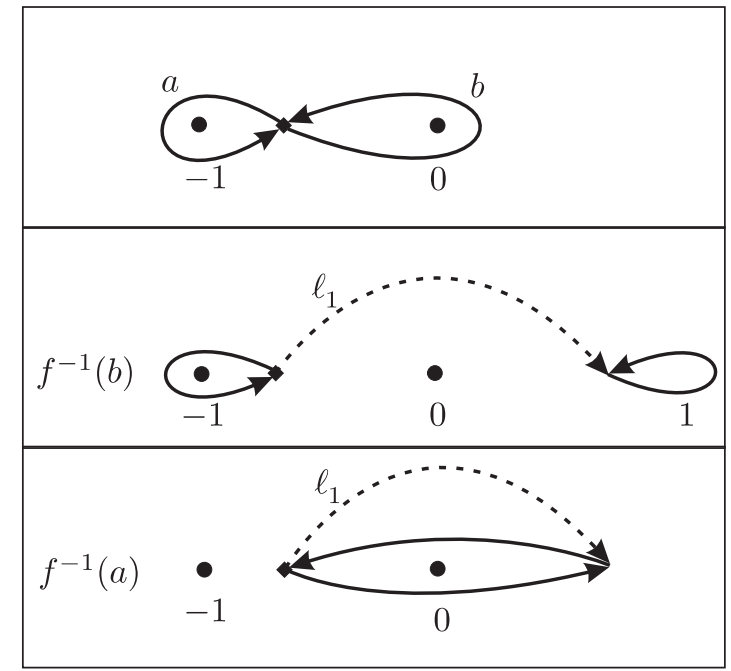

Figure 10. Computation of the group $\operatorname{IMG}\left(z^{2}-1\right)$

The preimages of the loops $a$ and $b$ are shown on the two lower parts of Figure 10. It follows that

$$
a \cdot x_{0}=b \cdot x_{1}, \quad a \cdot x_{1}=x_{0} \cdot 1, \quad b \cdot x_{0}=x_{0} \cdot a, \quad b \cdot x_{1}=x_{1} \cdot 1,
$$


so that the group IMG $\left(z^{2}-1\right)$ is generated by the automaton with the Moore diagram shown on Figure 11.

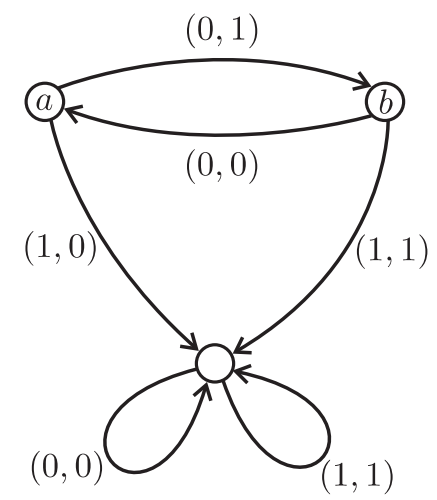

Figure 11. Automaton generating $\operatorname{IMG}\left(z^{2}-1\right)$

The group IMG $\left(z^{2}-1\right)$ was studied by R. Grigorchuk and A. Żuk in [GŻ02a, GŻ02b]. They defined it just as an interesting group generated by a three-state automaton. R. Pink discovered that it is the iterated monodromy group of $z^{2}-1$. More precisely, he defined the closed iterated monodromy groups as Galois groups (see below) and computed $\overline{\mathrm{IMG}}\left(z^{2}-1\right.$ ) using only the information about the conjugacy classes of $a_{1}, a_{2}$ and $a_{1} a_{2}$ in Aut $X^{*}$.

Theorem 4.4 (R. Grigorchuk, A. Żuk). The group $\operatorname{IMG}\left(z^{2}-1\right)$

(1) is torsion free;

(2) has exponential growth (actually, the semigroup generated by a and $b$ is free);

(3) is just non-solvable, i.e., every its proper quotient is solvable;

(4) has solvable word and conjugacy problems;

(5) has no free non-abelian subgroups of rank 2;

(6) is not in the class $S G$ of subexponentially amenable groups.

The class $S G$, which is the smallest class containing groups of subexponential growth and closed under taking extensions and direct limits.

It was proved in [BV05] using self-similarity of random walks, that IMG $\left(z^{2}-1\right)$ is amenable. It is the first example of an amenable group not belonging to the class $S G$. 


\subsection{Iterated monodromy groups of rational functions}

Let $f(z) \in \mathbb{C}(z)$ be a rational function seen as a branched covering of the Riemann sphere $\widehat{\mathbb{C}}=\mathbb{C} \cup\{\infty\}$. If $p, q \in \mathbb{C}[z]$ are coprime polynomials such that $f(z)=p(z) / q(z)$, then the degree of $f$ is $\max (\operatorname{deg} p, \operatorname{deg} q)$. The degree of $f$ is equal to the topological degree of the branched covering.

Let $C_{f}$ be the set of critical points of $f$. We denote by $P_{f}$ the set of post-critical points of $f$, i.e., the union

$$
P_{f}=\bigcup_{n \geq 1} f^{n}\left(C_{f}\right)
$$

of the forward orbits of the critical values. Here and below $f^{n}=f^{\circ n}$ denotes the $n$th iteration of $f$ rather than the $n$th degree.

If $P_{f}$ is finite, then the rational function $f$ is called post-critically finite. In this case $f$ is a partial self-covering $f: \mathcal{M}_{1} \longrightarrow \mathcal{M}$ for $\mathcal{M}=\widehat{\mathbb{C}} \backslash P_{f}$ and $\mathcal{M}_{1}=\widehat{\mathbb{C}} \backslash f^{-1}\left(P_{f}\right)$. The sets $\mathcal{M}$ and $\mathcal{M}_{1}$ are punctured spheres. The fundamental group $\pi_{1}(\mathcal{M})$ is the free group of rank $\left|P_{f}\right|-1$.

Definition 4.1. The iterated monodromy group of a post-critically finite rational function $f(z)$ is the iterated monodromy group of the partial selfcovering $f: \mathcal{M}_{1} \longrightarrow \mathcal{M}$, where $\mathcal{M}=\widehat{\mathbb{C}} \backslash P_{f}$ and $\mathcal{M}_{1}=f^{-1}(\mathcal{M})$.

The following construction belongs to R. Pink (private communication).

Let $f(z) \in \mathbb{C}(z)$ be a rational function. Let $p_{n}(z), q_{n}(z) \in \mathbb{C}[z]$ be the coprime polynomials such that $p_{n}(z) / q_{n}(z)$ is the $n$th iteration $f^{n}$ of the rational function $f$. Consider the field $\Omega_{n}$ obtained by adjoining all the solutions of the equation $f^{n}(z)=t$ in an algebraic completion $\overline{\mathbb{C}(t)}$ to the field of rational functions $\mathbb{C}(t)$. In other words, $\Omega_{n}$ is the splitting field of the polynomial $F_{n}(z)=p_{n}(z)-q_{n}(z) t \in \mathbb{C}(t)[z]$ over the function field $\mathbb{C}(t)$. It is easy to see that $\Omega_{n} \subset \Omega_{n+1}$. It is well known that the Galois group $\operatorname{Aut}\left(\Omega_{n} / \mathbb{C}(t)\right)$ is isomorphic to the monodromy group of the branched covering $f^{n}: \mathbb{C} \longrightarrow \mathbb{C}$ (see, for example [For81] Theorem 8.12).

As a corollary, we get the following interpretation of the closed iterated monodromy group of a polynomial.

Proposition 4.5. Let $f \in \mathbb{C}(z)$ be a post-critically finite rational function. Then the closed iterated monodromy group $\overline{\operatorname{IMG}}(f)$ is isomorphic to the Galois group Aut $(\Omega / \mathbb{C}(t))$, where $\Omega=\bigcup_{n \geq 1} \Omega_{n}$. 


\section{Contracting actions and limit spaces}

\subsection{Contracting actions}

Definition 5.1. A self-similar action $(G, \mathrm{X})$ is called contracting if there exists a finite set $\mathcal{N} \subset G$ such that for every $g \in G$ there exists $k \in \mathbb{N}$ such that $\left.g\right|_{v} \in \mathcal{N}$ for all the words $v \in \mathrm{X}^{*}$ of length $\geq k$. The minimal set $\mathcal{N}$ with this property is called the nucleus of the self-similar action.

If $A, B$ are subsets of the group $G$, then we denote by $A \cdot B$ the set $\{a b: a \in A, b \in B\} \subset G$ and by $\left.A\right|_{v}\left(v \in \mathrm{X}^{*}\right)$ we denote the set of the restrictions $\left\{\left.a\right|_{v}: a \in A\right\}$. We also write $A^{k}$ as a short notation for $\underbrace{A \cdot A \cdots A}_{k}$.

Lemma 5.1. A self-similar action $(G, \mathrm{X})$ where $G$ is a group generated by a finite set $S=S^{-1}, 1 \in S$, is contracting if and only if there exists a finite set $\mathcal{N}$ and a number $k \in \mathbb{N}$ such that for every word $v \in \mathrm{X}^{*}$ of the length greater than $k$ we have

$$
\left.\left((S \cup \mathcal{N})^{2}\right)\right|_{v} \subseteq \mathcal{N} .
$$

Proof. Induction on length of the group's element using equations (6).

It follows that there is an algorithm, which given a self-similar action of a finitely-generated group, stops if and only if the action is contracting. It is also not hard to see that there exists an algorithm, which given a contracting action gives its nucleus.

As an example of a contracting action, one can take the adding machine action of the group $\mathbb{Z}$. If we take $S=\{-1,0,1\}$ then $2 S=\{-2,-1,0,1,2\}$. The restrictions of the elements of $2 S$ in the words of length $>1$ are $\{-1,0,1\}$, so the nucleus is the set $\{-1,0,1\}$.

Other examples of contracting actions include the Grigorchuk group and the Gupta-Sidki group. The contraction of the Grigorchuk group was used in the original paper [Gri80] to prove that every its element has a finite order. The nucleus of the Grigorchuk group coincides with the automaton defining the generators and is shown on Figure 2. The lamplighter group and the free groups described in 2.9.4 are examples of finite-state but noncontracting actions.

It follows from Definition 5.1 that restrictions of the elements of the nucleus also belong to the nucleus. Thus the nucleus is a subautomaton of the complete automaton of the action. So we will consider the nucleus of a contracting action as an automaton, rather than just a subset of the group. For instance, the nucleus of the adding machine has the diagram shown on Figure 12. 


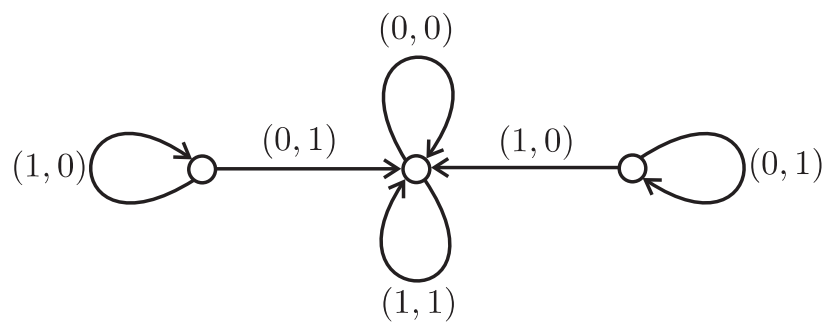

Figure 12. The nucleus of the adding machine action

Proposition 5.2. Suppose that a self-similar action of a finitely generated group $G$ is recurrent and contracting with the nucleus $\mathcal{N}$. Then $G=\langle\mathcal{N}\rangle$.

Recall that an action is said to be recurrent if the associated virtual endomorphism is onto, or, equivalently, if the left action of the associated bimodule is transitive.

Proof. Let $S$ be a finite generating set of the group $G$ and let $\phi$ be the virtual endomorphism associated with the self-similar action. There exist $n$ such that for every $g \in S$ and $v \in \mathrm{X}^{n}$ the restriction $\left.g\right|_{v}$ belongs to $\mathcal{N}$. Then the restriction of any element of $G$ in any word of length $n$ is a product of the elements of $\mathcal{N}$. Consequently, the range of $\phi^{n}$ belongs to the subgroup generated by $\mathcal{N}$. But the action is recurrent, so the range of $\phi^{n}$ is equal to $G$, and $G$ is generated by $\mathcal{N}$.

The following proposition is proved in [Nek05] (Corollary 2.11.7).

Proposition 5.3. Suppose that the self-similar action $(G, \mathrm{X})$ associated to a bimodule $\mathfrak{M}$ and a basis $\mathrm{X}$ is contracting. Let $\mathrm{Y}$ be another basis of $\mathfrak{M}$. Then the action $(G, \mathrm{Y})$ is also contracting and the conjugating transformation $\alpha$, defined in Proposition 3.3 is finite-state.

\subsection{Contraction coefficient}

Perhaps a more natural definition of a contracting action can be given when the group $G$ is finitely generated. Then contraction $\mathrm{f}$ the action is equivalent to contraction of length of the group elements under restrictions.

If $G$ is a group generated by a finite set $S=S^{-1}$ then we denote by $l(g)$ the word length of the group element $g \in G$, i.e., the minimal length of a representation of $g$ as a product of the elements of $S$. 
Definition 5.2. Let $(G, \mathrm{X})$ be a self-similar action of a finitely generated group. The number

$$
\rho=\limsup _{n \rightarrow \infty} \sqrt[n]{\limsup _{l(g) \rightarrow \infty} \max _{v \in \mathrm{X}^{n}} \frac{l\left(\left.g\right|_{v}\right)}{l(g)}}
$$

is called the contraction coefficient of the action.

Let $\phi$ be a virtual endomorphism of the group $G$. The number

$$
\rho_{\phi}=\limsup _{n \rightarrow \infty} \sqrt[n]{\limsup _{g \in \operatorname{Dom} \phi^{n}, l(g) \rightarrow \infty} \frac{l\left(\phi^{n}(g)\right)}{l(g)}},
$$

is called the contraction coefficient (or the spectral radius) of the virtual endomorphism $\phi$.

The numbers $\rho$ and $\rho_{\phi}$ are always finite since they are not greater than $\max _{g \in S, x \in \mathrm{X}} l\left(\left.g\right|_{x}\right)$, where $S$ is the generating set. It is easy to prove that $\rho$ and $\rho_{\phi}$ do not depend on the choice of the generating set.

The following proposition is proved in [Nek05] (Proposition 2.11.11).

Proposition 5.4. The action is contracting if and only if its contraction coefficient $\rho$ is less than one.

Let the action be level-transitive. If it is contracting, then $\rho=\rho_{\phi}<1$. If $\rho<1$ or $\rho_{\phi}<1$, then the action is contracting.

\subsection{Limit space $\mathfrak{J}_{G}$}

Let us fix a self-similar contracting action $(G, \mathrm{X})$. We consider the space $\mathrm{X}^{-\omega}=\left\{\ldots x_{2} x_{1}: x_{i} \in \mathrm{X}\right\}$ of the left-infinite sequences over the alphabet $X$. The space $\mathbf{X}^{-\omega}$ is an infinite direct power of the discrete space $\mathbf{X}$ and is obviously homeomorphic to the space $X^{\omega}$.

We say that a sequence $x_{1}, x_{2}, \ldots$ of elements of some set is bounded if the set of values $\left\{x_{i}\right\}$ of the sequence is finite.

Definition 5.3. Two elements $\ldots x_{3} x_{2} x_{1}, \ldots y_{3} y_{2} y_{1} \in \mathbf{X}^{-\omega}$ are said to be asymptotically equivalent with respect to the action $(G, \mathrm{X})$, if there exist a bounded sequence $g_{k} \in G, k \in \mathbb{N}$ such that

$$
g_{k}\left(x_{k} x_{k-1} \ldots x_{2} x_{1}\right)=y_{k} y_{k-1} \ldots y_{2} y_{1}
$$

for every $k \in \mathbb{N}$.

The following proposition gives a more convenient description of the asymptotic equivalence relation (see Theorem 3.6.3 in [Nek05]). 
Proposition 5.5. Let $\mathcal{N}$ be the nucleus of the action. Two sequences $\xi=\ldots x_{2} x_{1}, \zeta=\ldots y_{2} y_{1} \in \mathrm{X}^{-\omega}$ are asymptotically equivalent if and only if there exists a sequence $h_{n} \in \mathcal{N}, n \geq 0$ such that

$$
h_{n} \cdot x_{n}=y_{n} \cdot h_{n-1}
$$

for all $n \geq 1$.

Proposition 5.5 can be formulated in the following terms

Proposition 5.6. Let $\Gamma$ be the Moore diagram of the nucleus $\mathcal{N}$. Two sequences $\ldots x_{2} x_{1}, \ldots y_{2} y_{1} \in \mathbf{X}^{-\omega}$ are asymptotically equivalent if and only if the Moore diagram $\Gamma$ has a path $\left(\ldots, e_{2}, e_{1}\right)$ such that every edge $e_{i}$ of the path is labeled by the pair $\left(x_{i}, y_{i}\right)$.

Definition 5.4. The limit space of a self-similar action (denoted $\mathcal{J}_{G}$ ) is the quotient of the topological space $X^{-\omega}$ by the asymptotic equivalence relation.

It follows from the definition that the asymptotic equivalence relation is invariant under the shift map $\sigma: \ldots x_{3} x_{2} x_{1} \mapsto \ldots x_{4} x_{3} x_{2}$, and thus the shift $\sigma: \mathbf{X}^{-\omega} \rightarrow \mathbf{X}^{-\omega}$ induces a surjective continuous map s : $\mathcal{J}_{G} \rightarrow \mathcal{J}_{G}$ on the limit space $\mathcal{J}_{G}$. Every point $\xi \in \mathcal{J}_{G}$ has not more than $|\mathrm{X}|$ preimages under $\mathrm{s}$.

Definition 5.5. The dynamical system $\left(\mathcal{J}_{G}, \mathrm{~s}\right)$ is called the limit dynamical system of the self-similar action.

Example. Consider the adding machine action of $\mathbb{Z}$. Then one sees on the diagram of the nucleus (Figure 12) that two sequences are asymptotically equivalent if and only if they are either equal or are of the form

or of the form

$$
\ldots 0001 x_{m} x_{m-1} \ldots x_{1} \quad \ldots 1110 x_{m} x_{m-1} \ldots x_{1},
$$

$$
\ldots 000 \quad \ldots 111 \text {, }
$$

here $x_{m} x_{m-1} \ldots x_{1} \in \mathrm{X}^{*}$ is an arbitrary finite (possibly empty) word.

But this is the usual identification of the dyadic expansions of reals

$$
0 . x_{1} x_{2} \ldots x_{m} 0111 \ldots=0 . x_{1} x_{2} \ldots x_{m} 1000 \ldots,
$$

more precisely, two sequences $\ldots x_{2} x_{1}, \ldots y_{2} y_{1}$ are equivalent if and only if

$$
\sum_{n=1}^{\infty} x_{n} \cdot 2^{-n}=\sum_{n=1}^{\infty} y_{n} \cdot 2^{-n} \quad(\bmod 1) .
$$

Consequently, the limit space $\mathcal{J}_{\mathbb{Z}}$ is the circle $\mathbb{R} / \mathbb{Z}$. The map $\mathrm{s}$ is the two-fold self-covering map $\mathrm{s}(x)=2 x(\bmod 1)$.

Proposition 5.5 implies the next properties of the limit spaces. 
Proposition 5.7. The limit space $\partial_{G}$ is metrizable and has topological dimension $\leq|\mathcal{N}|-1$, where $\mathcal{N}$ is the nucleus of the action.

Proof. It follows directly from Proposition 5.5 that the asymptotic equivalence relation is closed.

Every $h_{n}$ in Proposition 5.5 is uniquely defined by $x_{n+1}$ and $h_{n+1}$, so every asymptotical equivalence class has not more than $|\mathcal{N}|$ elements.

Now by Theorem 4.2.13 from [Eng77], the quotient space $\mathcal{J}_{G}$ is metrizable, since it is a quotient of a compact separable metrizable space $\mathbf{X}^{-\omega}$ by a closed equivalence relation with compact equivalence classes. The assertion about the dimension follows from the fact that the space $\mathbf{X}^{-\omega}$ is 0 -dimensional and that every equivalence class is of cardinality $\leq|\mathcal{N}|$, due to the Hurewicz formula (see [Kur61] page 52).

Proposition 5.8. If the action $(G, \mathrm{X})$ is finitely-generated and level-transitive, then the space $\mathcal{J}_{G}$ is connected. If, additionally, the action is recurrent, then the space $\mathcal{J}_{G}$ is locally connected.

(See Theorem 3.6.3 of [Nek05].)

\subsection{Limit $G$-space $X_{G}$}

Let $(G, \mathrm{X})$ be a contracting self-similar action and let $\mathfrak{M}=\mathrm{X} \cdot G$ be the associated bimodule. The boundary $X^{\omega}$ of the tree $X^{*}$ can be naturally interpreted as an infinite tensor power $\mathfrak{M}^{\otimes \omega}=\mathfrak{M} \otimes \mathfrak{M} \otimes \cdots$, which has an obvious structure of a left $G$-module.

We want to show here that there exists a nice notion of a left-infinite tensor power

$$
\mathfrak{M}^{\otimes-\omega}=\cdots \otimes \mathfrak{M} \otimes \mathfrak{M}
$$

which is a right $G$-module and that the limit space $\mathcal{J}_{G}$ is the space of orbits of the action of $G$ on $\mathfrak{M}^{\otimes-\omega}$. In this sense the limit space $\mathcal{J}_{G}$ becomes a sort of dual of the self-similar action on $X^{\omega}$.

Let $\Omega(\mathfrak{M})$ be the set of all formal expressions $\ldots \otimes x_{2} \otimes x_{1}$, where $x_{1}, x_{2}, \ldots$ is a bounded sequence of elements $x_{i} \in \mathfrak{M}$. We have

$$
\Omega(\mathfrak{M})=\bigcup_{\mathrm{Y} \subset \mathfrak{M},|Y|<\infty} \mathrm{Y}^{-\omega},
$$

and we take $\Omega(\mathfrak{M})$ with the direct limit topology given by this decomposition into a union.

Definition 5.6. Two sequences $\ldots \otimes x_{2} \otimes x_{1}, \ldots \otimes y_{2} \otimes y_{1} \in \Omega(\mathfrak{M})$ are asymptotically equivalent if there exists a bounded sequence $g_{n} \in G$ such that

$$
g_{n} \cdot x_{n} \otimes x_{n-1} \otimes \cdots \otimes x_{1}=y_{n} \otimes y_{n-1} \otimes \cdots \otimes y_{1}
$$


in $\mathfrak{M}^{\otimes n}$ for every $n \geq 1$.

The quotient of space $\Omega(\mathfrak{M})$ by the asymptotic equivalence relation is denoted $\mathfrak{M}^{\otimes-\omega}$, or $X_{G}$ and is called the limit $G$-space.

It is easy to see that the space $\mathfrak{M}^{\otimes-\omega}$ is a right $G$-space, i.e., that the right action

$$
\left(\ldots \otimes x_{2} \otimes x_{1}\right) \cdot g=\ldots \otimes x_{2} \otimes\left(x_{1} \cdot g\right)
$$

is a well defined action on $\mathfrak{M}^{\otimes-\omega}$. We will write the sequence $\ldots \otimes x_{2} \otimes x_{1}$ usually just as a left-infinite word $\ldots x_{2} x_{1}$.

One can show (see [Nek05]) that for any basis $\mathbf{X}$ of $\mathfrak{M}$ we can write every element $\ldots a_{2} a_{1} \in \mathfrak{M}^{\otimes-\omega}$ in the form $\ldots x_{2} x_{1} \cdot g$ for some $x_{i} \in \mathrm{X}$ and $g \in G$.

Let us denote by $\mathrm{X}^{-\omega} \cdot G$ the set of sequences $\ldots x_{2} x_{1} \cdot g$ for $x_{i} \in \mathrm{X}$ and $g \in G$. We introduce the direct product topology on it, where $\mathrm{X}$ and $G$ are discrete. We get then the following (Proposition 3.2.6 of [Nek05]).

Theorem 5.9. Two elements $\ldots x_{2} x_{1} \cdot g$ and $\ldots y_{2} y_{1} \cdot h$ of $\mathrm{X}^{-\omega} \cdot G$ are asymptotically equivalent if and only if there exists a left-infinite directed path $\ldots e_{2} e_{1}$ in the Moore diagram of the nucleus $\mathcal{N}$ ending in the vertex $\mathrm{hg}^{-1}$ such that the edge $e_{i}$ is labeled by $\left(x_{i}, y_{i}\right)$.

The quotient of $\mathrm{X}^{-\omega} \cdot G \subset \Omega(\mathfrak{M})$ by the asymptotic equivalence relation is homeomorphic to $X_{G}$.

Example. In the case of the adding machine action of $\mathbb{Z}=\langle a\rangle$ one sees on the diagram of the nucleus (Figure 12 on page 72) that two sequences are asymptotically equivalent if and only if they are either equal or are of the form

$$
\ldots 0001 x_{m} x_{m-1} \ldots x_{1} \cdot a^{n} \quad \ldots 1110 x_{m} x_{m-1} \ldots x_{1} \cdot a^{n},
$$

where $x_{m} x_{m-1} \ldots x_{1} \in \mathrm{X}^{*}$ is an arbitrary finite (possibly empty) word, or of the form

$$
\ldots 000 \cdot a^{n+1} \quad \ldots 111 \cdot a^{n} .
$$

But this is the usual identification of the dyadic expansions of reals

$$
n . x_{1} x_{2} \ldots x_{m} 0111 \ldots=n . x_{1} x_{2} \ldots x_{m} 1000 \ldots,
$$

i.e., two sequences $\ldots x_{2} x_{1} \cdot a^{n}, \ldots y_{2} y_{1} \cdot a^{m}$ are equivalent if and only if

$$
n+\sum_{i=1}^{\infty} x_{i} \cdot 2^{-i}=m+\sum_{i=1}^{\infty} y_{i} \cdot 2^{-i}
$$

Consequently, the limit space $X_{G}$ is the real line $\mathbb{R}$ with the natural action of $\mathbb{Z}$ on it. 


\subsection{Limit space $\partial_{G}$ as a quotient of $X_{G}$}

The action of $G$ on $X_{G}$ is defined in terms of $\mathrm{X}^{-\omega} \cdot G$ by the equality

$$
\left(\ldots x_{2} x_{1} \cdot g\right) \cdot h=\ldots x_{2} x_{1} \cdot g h .
$$

If we look at the definition of the asymptotic equivalence relation on $\mathrm{X}^{-\omega} \cdot G$ and on $\mathrm{X}^{-\omega}$, then we see that the quotient of the limit space $X_{G}$ by the action of $G$ is homeomorphic to the limit space $\mathcal{J}_{G}$, where the homeomorphism is induced by the projection map

$$
\ldots x_{2} x_{1} \cdot g \mapsto \ldots x_{2} x_{1} .
$$

The limit $G$-space $X_{G}$ was constructed initially using only the self-similarity bimodule $\mathfrak{M}$. Consequently, the limit space $\mathcal{J}_{G}=\mathcal{X}_{G} / G$ also depends only on the self-similarity bimodule.

A more detailed analysis shows that the following is true (see Theorem 4.6.4 of [Nek05]).

Theorem 5.10. Limit dynamical systems $\left(\mathcal{J}_{G}, \mathrm{~s}\right)$ of equivalent self-similar actions are topologically conjugate.

Topological properties of the limit space $x_{G}$ are similar to the properties of the limit space $\mathcal{J}_{G}$ : it is metrizable, locally compact and finitedimensional. It is connected if the group is finitely generated and the action is recurrent.

\subsection{Markov partition of $\left(\mathcal{J}_{G}, \mathrm{~s}\right)$}

Definition 5.7. For a given finite word $v \in \mathrm{X}^{*}$ define the tile $\mathcal{T}_{v} \subset \mathcal{J}_{G}$ to be equal to the image of the set $\mathrm{X}^{-\omega} v=\left\{\ldots x_{2} x_{1} v\right\}$ under the canonical $\operatorname{map} \mathrm{X}^{-\omega} \rightarrow \mathcal{J}_{G}$.

We denote by $J_{n}$ the set $\left\{\mathcal{T}_{v}: v \in \mathrm{X}^{n}\right\}$ (the set of the tiles of the $n t h$ level).

It follows from the definitions that $\mathcal{T}_{\emptyset}=\mathcal{J}_{G}$ and that

$$
\mathrm{s}\left(\mathcal{T}_{v y}\right)=\mathcal{T}_{v}=\bigcup_{x \in \mathrm{X}} \mathcal{T}_{x v}
$$

for all $v \in \mathbf{X}^{*}$ and $x, y \in \mathbf{X}$.

Consequently, for every fixed $n$ the set $J_{n}$ is a Markov partition of the dynamical system $\left(\mathcal{J}_{G}\right.$, s) , i.e., the image of an element of $J_{n}$ under the map $\mathrm{s}$ is a union of elements of $J_{n}$.

A definition of a Markov partition usually requires that the sets of the partition do not overlap, i.e., have disjoint interiors. This is not the case in general for the tiles $\mathcal{T}_{v} \in J_{n}$. For instance, if we take the self-similar 
action of the group $\mathbb{Z}$ defined by the virtual endomorphism $n \mapsto n / 2$ and the digit set $\{0,3\}$, then the limit space will be the circle $\mathbb{R} / \mathbb{Z}$ and the tiles $\mathcal{T}_{v} \in J_{n}$ will be the images of the sets $([0,3]+k) / 2^{n}$, where $k$ is an integer. See Section 6.6 of this paper for the general description of the limit spaces of Abelian groups, from which these statements follow. So in this case the tiles overlap, for instance $\left[0,3 / 2^{n}\right] \cap\left(\left[0,3 / 2^{n}\right]+1 / 2^{n}\right)=\left[1 / 2^{n}, 3 / 2^{n}\right]$. A similar example is presented in the paper [Vin95], where this problem for Abelian groups is discussed.

But there exists a simple criterion for the tiles to have disjoint interiors.

Definition 5.8. We say that a contracting action of a group $G$ satisfies the open set condition if for any element $g$ of the nucleus there exists a finite word $v \in \mathbf{X}^{*}$ such that $\left.g\right|_{v}=1$.

The next proposition follows from Proposition 3.3.7 of [Nek05].

Proposition 5.11. If the action satisfies the open set condition then every tile is closure of its interior and for every $n \geq 0$ the tiles from $J_{n}$ have disjoint interiors.

If the action does not satisfy the open set condition then for every $n$ big enough one can find a tile $\mathcal{T}_{v} \in J_{n}$ which is covered by the other tiles from $J_{n}$.

\subsection{Digit tiles of $x_{G}$}

Definition 5.9. The (digit) tile $\mathcal{T}=\mathfrak{T}(X)=\mathcal{T}(\mathfrak{M}, X)$ is the image of $X^{-\omega} \cdot 1$ in $X_{G}$, i.e., the set of points of $X_{G}$, which can be represented in the form $\ldots x_{2} x_{1}$ for $x_{i} \in \mathrm{X}$.

The following is a direct corollary of Theorem 5.9.

Proposition 5.12. Two sequences $\ldots x_{2} x_{1}, \ldots y_{2} y_{1} \in \mathrm{X}^{-\omega}$ represent the same point of the tile $\mathcal{T}(\mathrm{X})$ if and only if there exists a path ... $e_{2} e_{1}$ in the Moore diagram of the nucleus such that the arrow $e_{1}$ ends in the trivial state and every arrow $e_{i}$ is labeled by $\left(x_{i}, y_{i}\right)$.

The tile $\mathcal{T}(\mathbf{X})$ is homeomorphic to the quotient of the direct product $\mathbf{X}^{-\omega}$ by the described equivalence relation.

A tile $\mathcal{T}_{v}$ of $\mathcal{J}_{G}$ is a quotient of the space $\mathbf{X}^{-\omega}$ by the equivalence relation defined by paths in the Moore diagram of the nucleus which end in states $g$ stabilizing $v$, i.e., such that $g(v)=v$. Hence, a tile $\mathcal{T}_{v}$ is in general a continuous image of the digit tile $\mathcal{T}$.

We have

$$
x_{G}=\bigcup_{g \in G} \mathcal{T} \cdot g=\bigcup_{v \in \mathfrak{M}^{\otimes n}} \mathcal{T} \otimes v
$$


and

$$
\mathcal{T}=\bigcup_{v \in \mathrm{X}^{n}} \mathcal{T} \otimes v
$$

for every $n \in \mathbb{N}$.

The sets $\mathcal{T} \otimes v$ for $v \in \mathfrak{M}^{\otimes n}$ are called the tiles of $n$th level. The map $\xi \mapsto \xi \otimes v$ is a homeomorphism from $\mathcal{T}$ to $\mathcal{T} \otimes v$.

We have the following analog of Proposition 5.11.

Proposition 5.13. If the action satisfies the open set condition then the set

$$
D=\mathcal{T} \cap \bigcup_{g \in G, g \neq 1} \mathcal{T} \cdot g
$$

is equal to the boundary of $\mathcal{T}$, the set $\mathcal{T}$ is the closure of its interior and any two tiles of one level have disjoint interiors.

If the action does not satisfy the open set condition then $D=\mathcal{T}$ and every tile is covered by the other tiles of the same level.

It is not necessary to compute the nucleus of the action in order to know which sequences in $X^{-\omega}$ represent points of the boundary, as the following result says.

Proposition 5.14. Suppose that a contracting self-similar action $(G, \mathrm{X})$ satisfies the open set condition and is generated by a finite automaton $(\mathrm{A}, \mathrm{X})$. Then for every $\xi \in \partial \mathcal{T}$ there exists an oriented path $\ldots e_{2} e_{1}$ in the Moore diagram of $\mathrm{A}$ which ends in a non-trivial state of $\mathrm{A}$ and is labeled $\ldots\left(x_{2}, y_{2}\right)\left(x_{1}, y_{1}\right)$ where $\ldots x_{2} x_{1} \in \mathbf{X}^{-\omega}$ represents $\xi$.

\subsection{Adjacency of tiles}

The following is Proposition 3.6.8 and Proposition 3.3.5 of [Nek05].

Proposition 5.15. The tiles $\mathcal{T}_{v}, \mathcal{T}_{u}, u, v \in \mathrm{X}^{n}$ intersect if and only if there exists an element $h$ of the nucleus $\mathcal{N}$ such that $h(v)=u$.

The tiles $\mathcal{T} \otimes v, \mathcal{T} \otimes u, u, v \in \mathfrak{M}^{\otimes n}$ intersect if and only if there exists $h \in \mathcal{N}$ such that $h \cdot v=u$.

Denote by $\Gamma_{n}(G)$ the graph with the vertices identified with the tiles $\mathcal{T}_{v} \in J_{n}$ and two vertices connected by an edge if and only if the respective tiles have a nonempty intersection. This graph is called the tile adjacency graph of the $n$th level.

Definition 5.10. Let a group $G$ acting on a set $M$ be generated by a finite generating set $S$. Then the (simplicial) Schreier graph $\Gamma(G, S, M)$ of the action is the graph with the set of vertices $M$ in which two vertices $u, v \in M$ 
are adjacent if and only if one is obtained from the other by application of a generator $s \in S$.

Thus Proposition 5.15 can be formulated in the following way.

Corollary 5.16. The map $v \mapsto \mathcal{T}_{v}$ is an isomorphism of the Schreier graph $\Gamma\left(\langle\mathcal{N}\rangle, \mathcal{N}, \mathrm{X}^{n}\right)$ with the graph $\Gamma_{n}(G)$.

If the action is recurrent then, due to Proposition 5.2, the nucleus $\mathcal{N}$ generates the group $G$, and thus the graphs $\Gamma_{n}(G)$ are Schreier graphs of the group $G$.

The following theorem shows that the Schreier graphs are good approximations of the limit space $\mathcal{J}_{G}$ (it is Theorem 3.6.9 of [Nek05]).

Theorem 5.17. A compact Hausdorff space $\mathfrak{X}$ is homeomorphic to the limit space $\mathcal{J}_{G}$ if and only if there exists a collection $\mathfrak{T}=\left\{T_{v}: v \in \mathbf{X}^{*}\right\}$ of closed subsets of $\mathfrak{X}$ such that the following conditions hold.

(1) $T_{\emptyset}=\mathfrak{X}$ and $T_{v}=\cup_{x \in X} T_{x v}$ for every $v \in \mathrm{X}^{*}$.

(2) The set $\cap_{n=1}^{\infty} T_{x_{n} x_{n-1} \ldots x_{1}}$ contains only one point for every word $\ldots x_{2} x_{1} \in \mathrm{X}^{-\omega}$.

(3) The intersection $T_{v} \cap T_{u}$ for $u, v \in \mathrm{X}^{n}$ is non-empty if and only if there exists an element s of the nucleus of the group $G$ such that $s(v)=u$.

If $\mathfrak{X}$ is a metric space then condition (2) is equivalent to the condition

$$
\lim _{n \rightarrow \infty} \max _{v \in \mathbf{X}^{n}} \operatorname{diam}\left(T_{v}\right)=0 .
$$

\section{Examples and applications}

\subsection{Expanding self-coverings}

Let $\mathcal{M}$ be a Riemannian manifold and let $f: \mathcal{M}_{1} \longrightarrow \mathcal{M}$ be a smooth partial self-covering.

Definition 6.1. A map $f: \mathcal{M}_{1} \longrightarrow \mathcal{M}$, where $\mathcal{M}_{1}$ is an open subset of a Riemannian manifold $\mathcal{M}$, is expanding if there exist constants $C>0$ and $\lambda>1$ such that $\left\|D f^{n} \vec{v}\right\| \geq C \lambda^{n}\|\vec{v}\|$ for every non-zero tangent vector $\vec{v}$ to $\mathcal{M}_{n}$ and every $n$, where $\mathcal{M}_{n}$ is the domain of the $n$th iterate $f^{n}$ of $n$.

The Julia set of an expanding map $f$, denoted $\mathcal{J}(f)$, is the set of the accumulation points of $\bigcup_{n=0}^{\infty} f^{-n}\left(z_{0}\right)$, where $z_{0} \in \mathcal{M}$ is arbitrary.

It is not hard to prove that if the Julia set is compact and non-empty, then it does not depend on the choice of the point $z_{0}$ and $f\left(\mathcal{J}_{f}\right)=f^{-1}\left(\mathcal{J}_{f}\right)$ $=\mathcal{J}_{f}$. 
The following theorem is proved in [Nek05], Theorem 5.5.3 (see also [Nek02]).

Theorem 6.1. Let $f: \mathcal{M}_{1} \longrightarrow \mathcal{M}$ be an expanding partial self-covering map on $\mathcal{M}$. Suppose that $\mathcal{M}$ is complete and that the Julia set $\mathcal{J}_{f}$ is compact and nonempty. Then every standard action of $G=\operatorname{IMG}(f)$ on $\mathrm{X}^{*}$ is contracting and the limit dynamical system $\left(\mathcal{J}_{G}, \mathrm{~s}\right)$ is topologically conjugated to the dynamical system $\left(\mathcal{J}_{f}, f\right)$.

\subsection{Hyperbolic and sub-hyperbolic rational functions}

Let $f(z) \in \mathbb{C}(z)$ be a rational function. By definition, the Julia set of $f$ is the set of points $z \in \widehat{\mathbb{C}}=\mathbb{C} \cup\{\infty\}$ such that the sequence $\left\{f^{n}: n \in \mathbb{N}\right\}$ is not normal on any neighborhood of $z$ (see [Mil99]). Here, as usual $f^{n}$ denotes the $n$th iteration of $f$.

The set $\bigcup_{n \geq 0} f^{-n}(z)$ is dense in the Julia set $\mathcal{J}_{f}$ for every $z \in \mathcal{J}_{f}$ (see [Mil99], or [Lyu87]). Therefore our definition of the Julia set of an expanding map agrees with the classical notion.

Definition 6.2. A rational function $f \in \mathbb{C}(z)$ is said to be hyperbolic if it is expanding on a neighborhood of its Julia set.

We have the following criterion, originally due to Fatou (see [Fat20] and [Mil99] Theorem 19.1).

Theorem 6.2. A rational function $f \in \mathbb{C}(z)$ is hyperbolic if and only if the closure of the postcritical set $\overline{P_{f}}$ does not intersect the Julia set $\mathcal{J}_{f}$, or equivalently, if and only if the orbit of every critical point converges to an attracting cycle.

In particular, if every critical point belongs to a cycle, or is pre-periodic, but the period contains a critical point, then the rational function is hyperbolic.

A function is sub-hyperbolic if it is expanding respectively to some orbifold metric on a neighborhood of the Julia set. An analog of Theorem 6.2 is the following criterion (see [Mil99]).

Theorem 6.3. A rational function is sub-hyperbolic if and only if every orbit of a critical point is either finite, or converges to an attracting cycle.

In particular, every post-critically finite function is sub-hyperbolic. We get therefore the following theorem.

Theorem 6.4. Let $f \in \mathbb{C}(z)$ be a post-critically finite rational function. Then every standard self-similar action of the iterated monodromy group 
IMG $(f)$ is contracting and the limit dynamical system $\left(\mathcal{J}_{\mathrm{IMG}(f)}, \mathrm{s}\right)$ is topologically conjugate to the dynamical system $\left(\mathcal{J}_{f}, f\right)$. In particular, the limit space $\mathcal{J}_{\operatorname{IMG}(f)}$ is homeomorphic to the Julia set of $f$.

Theorem 6.4 follows directly from Theorem 6.1 if the function $f$ is hyperbolic. We have to use a version of Theorem 6.1 for orbi-spaces (see [Nek05]) in the general case of a sub-hyperbolic function.

Theorem 6.4 provides a finite-to-one encoding of the points of the Julia set $\mathcal{J}_{f}$ by infinite sequences, which semi-conjugates $f$ to the shift. This and similar encodings where constructed in [Yac73, Yac80, Guc70], see also [Lyu87] p. 81-82.

\subsection{Groups generated by bounded automata}

Let $(\mathrm{A}, \mathrm{X})$ be a finite automaton. Let us denote by $\alpha(k, q)$, for $k \in \mathbb{N}$ and $q \in \mathrm{A}$, the number of words $v \in \mathrm{X}^{n}$ such that $\left.q\right|_{v} \neq 1$.

S. Sidki studied the function $\alpha(k, q)$ in the paper [Sid00] (he used a different, but equivalent function).

Definition 6.3. A finite automaton $(A, X)$ is bounded if the sequence $\alpha(k, q)$ is bounded as a function of $k$ for each state $q \in \mathrm{A}$.

The set of all automorphisms of $\mathrm{X}^{*}$ defined by bounded automata is a group called the group of bounded automata. Let us denote it by $\mathcal{B}$.

We say that a self-similar action $(G, \mathrm{X})$ is an action by bounded automata if the set of automorphisms it defines on $X^{*}$ is a subgroup of $\mathcal{B}$.

The following theorem from [BN03] relates this notion with the geometry of the limit space $\mathcal{J}_{G}$.

Theorem 6.5. A self-similar finitely-generated group acting by bounded automata is always contracting.

A contracting finitely-generated self-similar group $(G, \mathrm{X})$ acts by bounded automata if and only if it satisfies the open set condition and the boundary of the tile $\mathcal{T} \subset X_{G}$ is a finite set.

If $(G, \mathrm{X})$ is a self-similar action by bounded automata, then the limit spaces $\mathcal{X}_{G}$ and $\mathcal{J}_{G}$ are of topological dimension 1.

An automorphism of the rooted tree $\mathbf{X}^{*}$ is said to be finitary if there exists $n \in \mathbb{N}$ such that $g\left(x_{1} x_{2} \ldots x_{m}\right)=g\left(x_{1} x_{2} \ldots x_{n}\right) x_{n+1} x_{n+2} \ldots x_{m}$, i.e., if $\left.g\right|_{v}=1$ for all $v \in X^{n}$. The set of finitary automorphisms is a locally finite subgroup of Aut $X^{*}$.

If $(G, \mathrm{X})$ is a self-similar action by bounded automata, then the Moore diagram of the complete automaton has only a finite number of oriented 
cycles of non-trivial states. These cycles have no common vertices and there is no directed path from a vertex of one cycle to a vertex of another.

If $g \in G$ belongs to a cycle of the Moore diagram of the complete automaton, then there exists precisely one letter $x \in \mathrm{X}$ such that $\left.g\right|_{x}$ belongs to a cycle. For all other letters $y \neq x$ the restriction $\left.g\right|_{y}$ is finitary.

The nucleus of a group generated by bounded automata consists of the states belonging to the cycles and their restrictions. In other words, the nucleus is equal to the set of elements $g \in G$ for which there exist $h \in G$ and $v, u \in \mathrm{X}^{*}$ such that $\left.h\right|_{v}=h$ and $\left.h\right|_{u}=g$.

Consequently, if $\ldots x_{2} x_{1} \in \mathrm{X}^{-\omega}$ represents a point of the boundary of the tile $\mathcal{T}$, then it is post-periodic, i.e., has the form $(v)^{-\omega} u$, where $v$ is a finite word read on the labels of a cycle, and $u$ is read on a path from a vertex of the cycle to a non-trivial state. Thus the set of words representing points of $\partial \mathcal{T}$ is finite and it is easy to find it (even without computing the nucleus, see Proposition 5.14).

\subsection{Tile diagrams}

There exists a simple iterative algorithm to draw approximations of the limit spaces $\mathcal{J}_{G}$ and tiles $\mathcal{T}$ of actions by bounded automata.

Let $(G, \mathrm{X})$ be a self-similar action by bounded automaton. Let $\mathcal{N}$ be the nucleus of the action.

We can find all sequences $\ldots x_{2} x_{1} \in \mathrm{X}^{-\omega}$ representing points of the boundary of the tile $\mathcal{T}$ as it is described above.

Thus, we can effectively find the points of the boundary $\partial \mathcal{T}$ finding all sequences encoding them and find out which points are glued together in $\mathcal{T}$ and $\mathcal{J}_{G}$.

Another technical condition which we need, but which is satisfied almost in all interesting cases, is connectedness of the tile $\mathcal{T}$.

The following criterion is a result of E. Bondarenko. It can be effectively used to determine if the tile $\mathcal{T}$ is connected.

Proposition 6.6. Let $T_{n}$ be the graph with the set of vertices $\mathbf{X}^{n}$ in which two vertices $v_{1}, v_{2}$ are connected by an edge if and only if there exists $h \in \mathcal{N}$ such that $h \cdot v_{1}=v_{2} \cdot 1$. Then the following conditions are equivalent

(1) The tile $\mathcal{T}$ is connected.

(2) The graphs $T_{n}$ are connected for all $n \geq 1$.

(3) The graph $T_{1}$ is connected.

We assume in this section that the tile $\mathcal{T}$ is connected. 
Definition 6.4. A tile diagram is a connected topological space $\Gamma$ together with a bijective correspondence between $\partial \mathcal{T}$ and a set of marked points of $\Gamma$.

If $\Gamma$ is a tile diagram, then its inflation $\Gamma \cdot \mathrm{X}$ is the tile diagram obtained by the following procedure

(1) Take $|\mathrm{X}|$ copies $\Gamma \cdot x$ of $\Gamma$. Here $x \in \mathrm{X}$ is a label, and if $v$ is a point of $\Gamma$, then $v \cdot x$ is the corresponding point of $\Gamma \cdot x$.

(2) Identify two points $v_{1} \cdot x_{1}$ and $v_{2} \cdot x_{2}$ if and only if $v_{1}$ and $v_{2}$ are marked in $\Gamma$ and the corresponding points $\xi_{1}, \xi_{2} \in \partial \mathcal{T}$ are such that $\xi_{1} \otimes x_{1}=\xi_{2} \otimes x_{2}$ in $X_{G}$.

(3) A point $v \cdot x$ is marked and corresponds to a point $\zeta \in \partial \mathcal{T}$ if and only if $v$ is marked in $\Gamma$ and $\zeta=\xi \otimes x$, where $\xi \in \partial \mathcal{T}$ is the point corresponding to $v$.

Connectedness of the tile $\mathcal{T}$ ensures that the inflation of a tile diagram is again connected.

The inflation $\Gamma \cdot \mathbf{X}$ can be easily computed using the nucleus and Theorem 5.9. Namely, if $v_{1}, v_{2} \in \Gamma$ are marked points corresponding to $\xi_{1}, \xi_{2}$, then the points $v \cdot x$ and $v \cdot y$ are identified in $\Gamma \cdot \mathrm{X}$ if and only if there exists a path $\gamma$ in the nucleus $\mathcal{N}$ of $(G, \mathrm{X})$ such that the left labels of the path $\gamma$ is a sequence representing $\xi_{1}$, right labels of $\gamma$ is a sequence representing $\xi_{2}$ and the end is a state $g \in \mathcal{N}$ such that $g \cdot x=y \cdot 1$. The point $v_{1} \cdot x$ is marked if and only if there exists a path $\gamma$ whose left labels is a path representing $\xi_{1}$ and which ends in a state $g \in \mathcal{N}$ such that $\left.g\right|_{x} \neq 1$.

We denote by $\Gamma \cdot \mathrm{X}^{n}$ the $n$th iteration of the inflation. The space $\Gamma \cdot \mathrm{X}^{n}$ consists of $|\mathrm{X}|^{n}$ pieces $\Gamma \cdot v, v \in \mathrm{X}^{n}$, glued together using the adjacency rule of tiles, described above.

If we rescale the spaces $\Gamma \cdot \mathrm{X}^{n}$ so that its pieces $\Gamma \cdot v$ become small, then the space $\Gamma \cdot \mathrm{X}^{n}$ will be a good approximation of the tile $\mathcal{T}$, due to Theorem 5.17.

We may for example consider only tile diagrams $\Gamma$ which are graphs such that the marked points are vertices. It is easy to see that inflation of a graph will be again a graph.

\section{Fabrikowski-Gupta group}

Consider an automaton over the alphabet $X=\{0,1,2\}$ with the set of states $\{a, b, 1\}$, given by

$$
a \cdot 0=1 \cdot 1, \quad a \cdot 1=2 \cdot 1, \quad a \cdot 2=0 \cdot 1
$$

and

$$
b \cdot 0=0 \cdot b, \quad b \cdot 1=1 \cdot a, \quad b \cdot 2=2 \cdot 1 .
$$


The group generated by this automaton was defined in [FG91]. The automaton is bounded, therefore the group $G$ generated by it acts by bounded automata.

The nucleus of the group $G$ is equal to $\left\{a, a^{-1}, 1, b, b^{-1}\right\}$. The only cycles of the Moore diagram are loops at the vertices $b$ and $b^{-1}$. These loops are labeled by $(0,0)$. It follows that the boundary of the tile $\mathcal{T}$ consists of two points represented by the sequences $0^{-\omega}$ and $0^{-\omega} 1$, respectively. They represent different points of the limit space $\mathcal{J}_{G}$, hence the tile $\mathcal{T}$ is homeomorphic to $\partial_{G}$.

We also see from the structure of the nucleus (from the action of $a$ ) that

$$
0^{-\omega} 10 \sim 0^{-\omega} 11 \sim 0^{-\omega} 12
$$

in $\mathcal{T}$.

Let us start from $\Gamma$ equal to a segment whose ends are marked and identified with $0^{-\omega}$ and $0^{-\omega} 1$, respectively.

Then identifications (23) implies that $\Gamma \cdot \mathbf{X}$ consists of three segments having one common end. Two endpoints of the obtained tripod are marked. Iterating the procedure, we get approximations of the limit space $J_{G}$. See for example the tile diagram $\Gamma \cdot X^{4}$ on Figure 13.

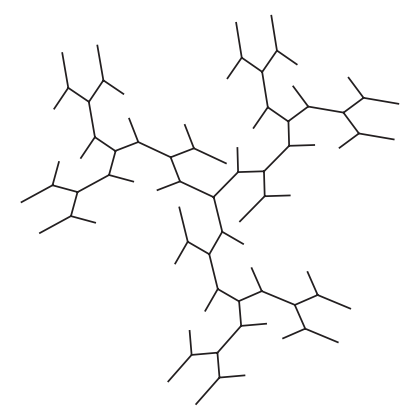

Figure 13. Tile diagram of Fabrikowski-Gupta group

\section{Basilica group}

Consider the group $\operatorname{IMG}\left(z^{2}-1\right)$. It is generated by the automaton shown of Figure 11.

The nucleus of IMG $\left(z^{2}-1\right)$ is equal to $\left\{1, a, b, a^{-1}, b^{-1}\right\}$. The boundary of the tile $\mathcal{T}$ is $\left\{0^{-\omega},(01)^{-\omega},(10)^{-\omega}\right\}$. All these points are glued together to one point in $\mathcal{J}_{G}$ but are different points in $\mathcal{T}$. We have also the following 
identifications of the points of $\mathcal{T}$ :

$$
0^{-\omega} 1 \sim(10)^{-\omega} 0 \sim(01)^{-\omega} 1 .
$$

If we start from the graph $\Gamma$ shown on the left-hand side part of Figure 14, then the right-hand side of the figure shows the graph $\Gamma \cdot X^{6}$. If we now identify the points $(10)^{-\omega}, 0^{-\omega}$ and $(01)^{-\omega}$, then we get an approximation of the limit space of the group IMG $\left(z^{2}-1\right)$, which is homeomorphic by Theorem 6.4 to the Julia set of the polynomial $z^{2}-1$. The point encoded by the sequences $0^{-\omega},(10)^{-\omega}$ and $(01)^{-\omega}$ is the fixed point $\frac{1-\sqrt{5}}{2}$ of the polynomial.

$(10)^{-}$

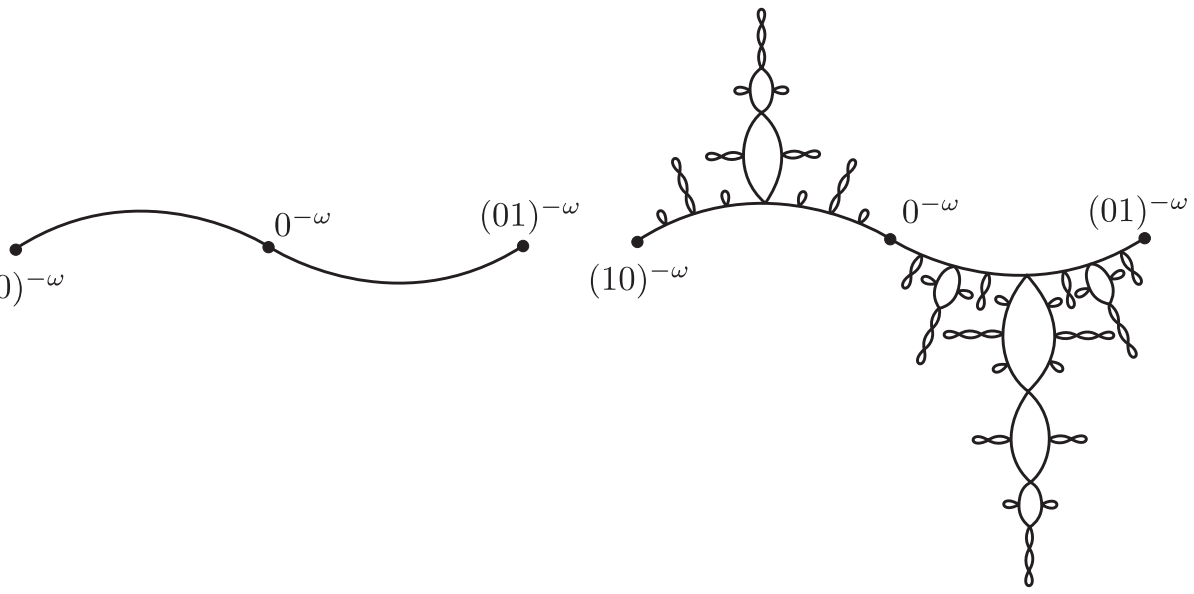

Figure 14. Basilica graphs

Compare the obtained graph with the Julia set of $z^{2}-1$ shown on Figure 15 .

\subsection{Iterated monodromy groups of polynomials}

Iterated monodromy groups of post-critically finite polynomials can be completely described in terms of automata that generate them.

Let $T$ be a subset of $\mathfrak{S}(\mathbf{X}) \backslash\{1\}$. Its cyclic diagram is the oriented 2-dimensional CW-complex whose set of 0 -cells is $\mathrm{X}$ and where for every non-trivial cycle $c=\left(x_{1}, x_{2}, \ldots, x_{k}\right)$ of every permutation $\pi \in T$ we have a 2-cell $C_{\pi, c}$ with vertices $x_{1}, x_{2}, \ldots, x_{n}$ so that their orders in the cycle and on the boundary of $C_{\pi, c}$ coincide. Different 2-cells intersect only along their 0-cells. 


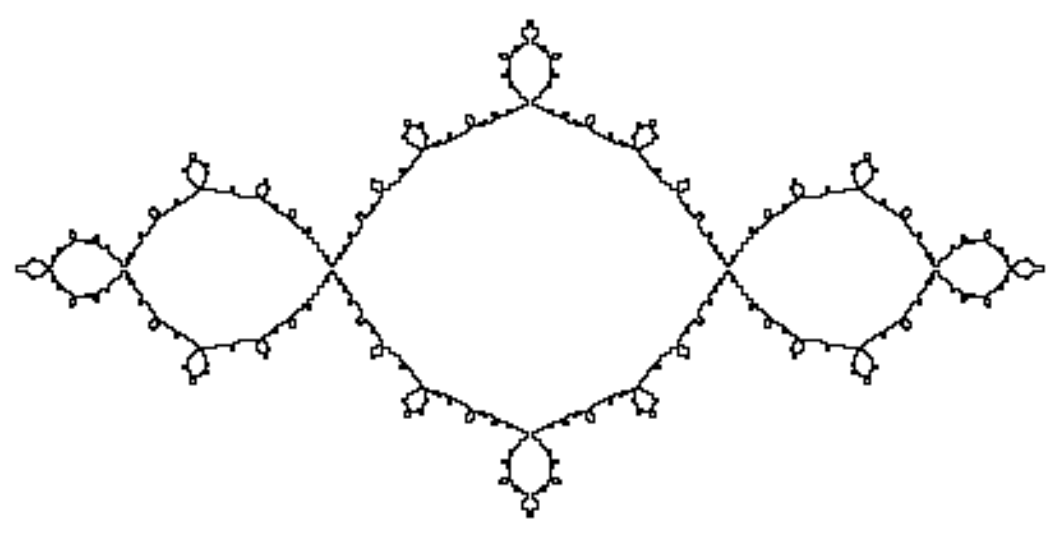

Figure 15. Julia set of $z^{2}-1$

Definition 6.5. A multi-set $T$ is said to be tree-like if its cyclic diagram is contractible.

For example, the only tree-like subset of non-trivial elements of $\mathfrak{S}(2)$ consists of the transposition and a tree-like subset of non-trivial elements of $\mathfrak{S}$ (3) consists either of one 3-cycle or of two different transpositions.

Definition 6.6. A finite permutational automaton $(\mathrm{A}, \mathrm{X})$ is a kneading automaton if

(1) every non-trivial state $g$ of $\mathrm{A}$ has a unique incoming arrow, i.e., there exist a unique pair $h \in \mathrm{A}, x \in \mathrm{X}$ such that $g=\left.h\right|_{x}$;

(2) for every cycle $\left(x_{1}, x_{2}, \ldots, x_{m}\right)$ of the action of a state $g$ on $\mathrm{X}$, the state $\left.g\right|_{x_{i}}$ is trivial for all but perhaps one letter $x_{i}$.

(3) the set of non-trivial permutations defined by the states of $A$ on $X$ is tree-like;

The first condition implies that every kneading automaton is bounded. Proofs of the next two theorems appear in [Nek05].

Theorem 6.7. For every post-critically finite polynomial $f \in \mathbb{C}[z]$ there exists a kneading automaton $(\mathrm{A}, \mathrm{X})$ such that

(1) The group generated by A is isomorphic to IMG $(f)$.

(2) The group $G=\langle\mathrm{A}\rangle$ is contracting and the Julia set of $f$ is homeomorphic to the limit space $\mathcal{J}_{G}$. 
(3) If $f$ is hyperbolic (more generally, if $f$ has an invariant spider), then the action of $G$ on $\mathrm{X}^{*}$ coincides with a standard action of $\operatorname{IMG}(f)$.

The automaton $(\mathrm{A}, \mathrm{X})$ is constructed using external rays and critical portraits (see [Poi93]).

Definition 6.7. Let $(\mathrm{A}, \mathrm{X})$ be a kneading automaton and $d=|\mathrm{X}|$. The automaton is said to be planar if there exists an ordering $g_{1}, g_{2}, \ldots, g_{n}$ of all its non-trivial states such that if we compute the state $\left.\left(g_{1} g_{2} \cdots g_{n}\right)^{d}\right|_{x}$ by the definition of $\left(\mathrm{A}^{n}, \mathrm{X}\right)$, then we get a cyclic shift of the word $g_{1} g_{2} \cdots g_{n}$ (after discarding the trivial states).

If a kneading automaton is planar, then we can change the action of $\langle A\rangle$ to an equivalent one so that $g_{1} g_{2} \cdots g_{n}$ becomes the $d$-adic adding machine $a=(1,1, \ldots, 1, a) \sigma$, where $\sigma$ is the transitive cycle $(1,2, \ldots, d)$.

It is easy to see that for every kneading automaton $(A, X)$ there exists a number $k \in \mathbb{N}$ such that the automaton $\left(\mathrm{A}, \mathrm{X}^{k}\right)$ is planar (one can also prove that if $(A, X)$ is a kneading automaton, then $\left(A, X^{k}\right)$ is also a kneading automaton).

Definition 6.8. Let $(A, X)$ be an abstract kneading automaton and let $G=\langle\mathrm{A}\rangle$ be the group it generates. We say that A has bad isotropy groups if there exists a point $\xi \in \mathcal{J}_{G}$ that is periodic under iterations of the shift s: $\mathcal{J}_{G} \longrightarrow \mathcal{J}_{G}$ and such that there exist its preimages $\xi_{1}, \xi_{2} \in X_{G}$ such that $\xi_{i} \cdot g_{i}=\xi_{i}$ for two different non-trivial states $g_{1}, g_{2}$ of A.

Theorem 6.8. If a kneading automaton $(\mathrm{A}, \mathrm{X})$ is planar, then it generates a standard action of the iterated monodromy group of a post-critically finite branched covering of the plane $\mathbb{R}^{2}$.

If the automaton is planar and has no bad isotropy groups, then it generates a standard action of the iterated monodromy group of a post-critically finite polynomial of degree $d=|\mathrm{X}|$. The polynomial is defined uniquely, up to an affine conjugation by the automaton and the circular order of its non-trivial states $g_{1}, g_{2}, \ldots, g_{n}$, satisfying conditions of Definition 6.7.

If a kneading automaton has no bad isotropy groups, then it generates an automorphism group of $\mathrm{X}^{*}$ which is conjugate to a standard action of the iterated monodromy group of a post-critically finite polynomial.

For example, the automaton generating the Fabrikowski-Gupta group is a planar kneading automaton and has no bad isotropy group, therefore it generates a standard action of the iterated monodromy group of a cubic polynomial. This polynomial is $f(z)=z^{3}(\zeta-1)+1$, where $\zeta=-\frac{1}{2}+\frac{\sqrt{3}}{2} i$ is a root of unity of degree 3 . One can take 0 as a basepoint and connect 
it to its preimages by straight lines. Direct computation of the standard action shows that it coincides with the Fabrikowski-Gupta group.

Compare the Julia set of this polynomial on Figure 16 with the approximation of the limit space of the group on Figure 13.

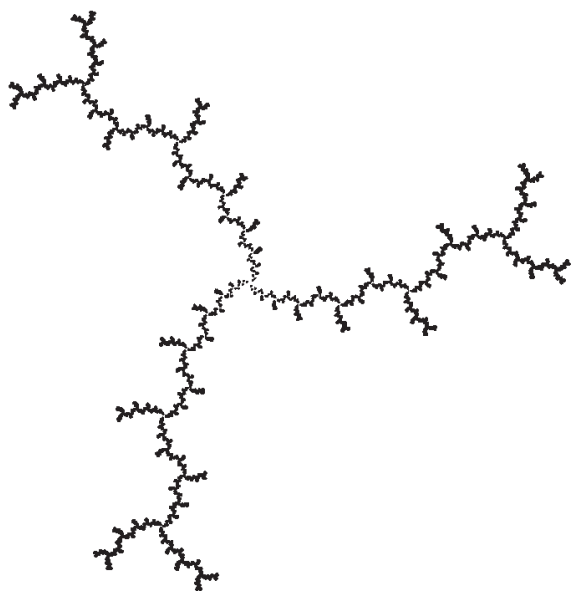

FigURE 16. Julia set of $z^{2}(-3 / 2+i \sqrt{3} / 2)+1$

\subsection{Abelian self-similar groups and their limit spaces}

Let $\left(\mathbb{Z}^{n}, \mathrm{X}\right)$ be a recurrent self-similar action of the free Abelian group. Let $\phi: \mathbb{Z}^{n} \rightarrow \mathbb{Z}^{n}$ be the associated virtual endomorphism and let $A$ : $\mathbb{Q}^{n} \longrightarrow \mathbb{Q}^{n}$ be its extension to a linear operator over $\mathbb{Q}$.

The following theorem is proved in [NS04] (for the case $|\mathrm{X}|=2$, but the proof easily extends to the general case).

Theorem 6.9. A self-similar action of the group $\mathbb{Z}^{n}$ is finite-state if and only if it is contracting, i.e., if the matrix A has spectral radius less than one.

Let $R=\left\{r_{0}=0, r_{1}, \ldots, r_{d-1}\right\}$ be a digit set of the action, i.e., such a set that $r_{i} \cdot x_{0}=x_{i} \cdot \overrightarrow{0}$, where $\mathbf{X}=\left\{x_{0}, x_{1}, \ldots, x_{d-1}\right\}$ and $\phi=\phi_{x_{0}}$ is defined with respect to $x_{0}$. Then $x_{i} \mapsto \phi\left(r_{i}\right)+\overrightarrow{0}$ induces an isomorphism of the self-similarity bimodule $\mathfrak{M}$ with the bimodule $\phi\left(\mathbb{Z}^{n}\right)+\mathbb{Z}^{n}$ and the self-similar action is defined by (15). 
For every sequence $w=i_{1} i_{2} \ldots \in \mathrm{X}^{\omega}$ the series

$$
F(w)=\sum_{k=1}^{+\infty} A^{k}\left(r_{i_{k}}\right)
$$

is convergent in $\mathbb{R}^{n}$. Let $\mathcal{T}=\mathcal{T}(\phi, R)=\left\{F(w): w \in \mathrm{X}^{\omega}\right\}$ be the set of all possible sums of such series. We will call $\mathcal{T}(\phi, R)$ the set of fractions, or the tile.

The set $\mathcal{T}(\phi, R)$ is the unique compact set invariant under the transformation

$$
P(C)=\bigcup_{i=0}^{d-1} p_{i}(C) .
$$

Moreover, for any nonempty compact set $C \subset \mathbb{R}^{n}$, the sequence $P^{n}(C)$ converges to $\mathcal{T}(\phi, R)$ with respect to the Hausdorff metric.

If $\operatorname{det} A=1 / 2$ and $A$ is the matrix of the associated virtual endomorphism, then $A$ is conjugate in $\mathrm{GL}(2, \mathbb{R})$ with one of the matrices

$$
\left(\begin{array}{cc}
0 & -\sqrt{2} / 2 \\
\sqrt{2} / 2 & 0
\end{array}\right), \quad\left(\begin{array}{cc}
1 / 4 & -\sqrt{7} / 4 \\
\sqrt{7} / 4 & 1 / 4
\end{array}\right), \quad\left(\begin{array}{cc}
-1 / 2 & -1 / 2 \\
-1 / 2 & 1 / 2
\end{array}\right),
$$

(see [NS04]).

For the case of a recurrent action on a binary tree (i.e., $|\mathrm{X}|=2$ ) the digit tile $\mathcal{T}(A, R)$ depends, up to an affine transformation, only on the conjugacy class of $A$ in $\mathrm{GL}(n, \mathbb{R})$ (see Proposition 6.2.1 of [Nek05]). This is not true for actions on trees of higher degree.

A tile of the actions defined by the virtual endomorphism $\left(\begin{array}{cc}0 & -\sqrt{2} / 2 \\ \sqrt{2} / 2 & 0\end{array}\right)$ is the "A4-paper" rectangle, i.e., the rectangle with sides of length $1 / \sqrt[4]{2}$ and $\sqrt[4]{2}$. If we take the conjugate matrix $\left(\begin{array}{cc}0 & 1 \\ 1 / 2 & 0\end{array}\right)$ and $R=\{(0,0),(1,0)\}$, then the set of fractions is the square $[0,1] \times[0,1]$.

The tile corresponding to the matrix $\left(\begin{array}{c}-1 / 2-1 / 2 \\ 1 / 2-1 / 2\end{array}\right)$ is the "twin dragon" shown on the left-hand side part of Figure 17. The twin dragon corresponds, for example to the self-similar action defined by the "binary numeration system" on the Gaussian integers with the base $(-1+i)$ and the digit set $\{0,1\}$. See its discussion in [Knu69].

The tile corresponding to the matrix $\left(\begin{array}{cc}1 / 4 & -\sqrt{7} / 4 \\ \sqrt{7} / 4 & 1 / 4\end{array}\right)$ is the "tame twin dragon" shown on the right-hand side of Figure 17.

See the works [Ban91, Vin95, Vin00] and bibliography therein for other examples of such sets and for their properties. These sets are used in 

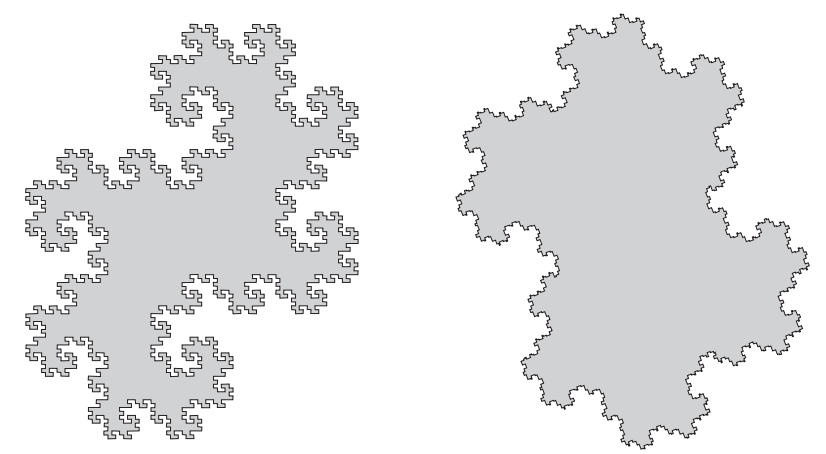

Figure 17. Twin dragon and tame twin dragon

the wavelet theory, computer image processing, toral dynamical systems and other fields. See, for example, the book [BJ99] for relations with $C^{*}$ algebras.

Now we are going to prove that the notion of a set of fractions in terms of the sums of series in $\mathbb{R}^{n}$ coincides with the general notion of a digit tile in $X_{\mathbb{Z}^{n}}$, defined in terms of asymptotically equivalent sequences.

Proposition 6.10. Two sequences $\left(\ldots x_{2} x_{1}\right)+g_{1}$ and $\left(\ldots y_{2} y_{1}\right)+g_{2} \in$ $\mathrm{X}^{-\omega}+\mathbb{Z}^{n}$ are asymptotically equivalent if and only if

$$
\sum_{k=1}^{+\infty} A^{k}\left(r_{x_{k}}\right)+g_{1}=\sum_{k=1}^{+\infty} A^{k}\left(r_{y_{k}}\right)+g_{2} .
$$

Proof. Suppose that the sequences $\left(\ldots x_{2} x_{1}\right)+g_{1}$ and $\left(\ldots y_{2} y_{1}\right)+g_{2}$ are asymptotically equivalent. Then there exists a sequence $s_{m}$ of elements of the nucleus of the action such that

$$
s_{m} \cdot\left(x_{m} \ldots x_{1}+g_{1}\right)=y_{m} \ldots y_{1}+g_{2} .
$$

It follows now from (15) on page 60 that

$$
\begin{aligned}
A^{m}\left(s_{m}\right)+\left(A^{m}\left(r_{x_{m}}\right)+\right. & \left.A^{m-1}\left(r_{x_{m-1}}\right)+\cdots+A\left(r_{x_{1}}\right)\right)+g_{1}= \\
& \left(A^{m}\left(r_{y_{m}}\right)+A^{m-1}\left(r_{y_{m-1}}\right)+\cdots+A\left(r_{y_{1}}\right)\right)+g_{2}
\end{aligned}
$$

Passing to the limit when $m \rightarrow \infty$, we get (24). 
Suppose now that (24) holds. Let us prove that the sequences are asymptotically equivalent. Define for every positive integer $m$

$$
\begin{aligned}
s_{m}=\left(r_{y_{m}}+A^{-1}\left(r_{y_{m-1}}\right)+\cdots+A^{-m+1}\left(r_{y_{1}}\right)+A^{-m}\left(g_{2}\right)\right)- \\
\left(r_{x_{m}}+A^{-1}\left(r_{x_{m-1}}\right)+\cdots+A^{-m+1}\left(r_{x_{1}}\right)+A^{-m}\left(g_{1}\right)\right) .
\end{aligned}
$$

Every $s_{m}$ belongs to $\mathbb{Z}^{n}$. It follows from (24) that

$$
s_{m}=\sum_{k=m+1}^{+\infty} A^{k-m}\left(r_{x_{k}}\right)-\sum_{k=m+1}^{+\infty} A^{k-m}\left(r_{y_{k}}\right) .
$$

Since $A$ has spectral radius less than one, we have a uniform upper bound on the norm of $s_{m} \in \mathbb{R}^{n}$. Therefore the set of all possible values of $s_{m}$ is finite and thus the sequences are asymptotically equivalent.

Theorem 6.11. Suppose that $\left(\mathbb{Z}^{n}, \mathrm{X}\right)$ is a contracting recurrent self-similar action and $\phi$ is the associated virtual endomorphism of $\mathbb{Z}^{n}$. Then

1) The limit space $X_{G}$ is homeomorphic to $\mathbb{R}^{n}$ with the natural action of $\mathbb{Z}^{n}$ on it.

2) The limit space $\mathcal{J}_{\mathbb{Z}^{n}}$ is homeomorphic to the torus $\mathbb{T}^{n}=\mathbb{R}^{n} / \mathbb{Z}^{n}$. There exists a homeomorphism which conjugates the shift $\mathrm{s}: \mathcal{J}_{\mathbb{Z}^{n}} \longrightarrow \mathcal{J}_{\mathbb{Z}^{n}}$ with the self-covering of the torus, induced by the linear transformation $A^{-1}=\phi^{-1}$.

First Proof. Let $A$ be the matrix of the virtual endomorphism $\phi$. Then the matrix $A^{-1}$ has integral entries and induces a $\left\|\operatorname{det}\left(A^{-1}\right)\right\|$-fold covering of the torus $\mathbb{R}^{n} / \mathbb{Z}^{n}$. This covering is expanding, since $A^{-1}$ is expanding on $\mathbb{R}^{n}$. The virtual endomorphism associated with the self-covering is obviously equal to $\phi$. Hence Theorem 6.1 implies the second statement of our theorem.

Let us prove the theorem directly, showing a connection between the construction of the limit space $X_{G}$ and numeration systems on $\mathbb{R}^{n}$.

Second Proof. For every $\xi \in \ldots x_{2} x_{1}+g \in \mathbf{X}^{-\omega}+\mathbb{Z}^{n}$, the sum of the series

$$
\Phi(\xi)=g+\sum_{k=1}^{+\infty} A^{k}\left(r_{y_{k}}\right)
$$

is convergent and the sum belongs to $\mathbb{R}^{n}$. Proposition 6.10 says that two sequences $\xi$ and $\zeta \in \mathrm{X}^{-\omega}+\mathbb{Z}^{n}$ represent the same point of $X_{\mathbb{Z}^{n}}$ if and only if $\Phi(\xi)=\Phi(\zeta)$. Therefore $\Phi$ induces a well defined injective map from $\mathcal{X}_{\mathbb{Z}^{n}}$ to $\mathbb{R}^{n}$. The contractivity of $A$ implies that the map is continuous. Let us prove that it is onto. 
Denote $B=[0,1]^{n} \subset \mathbb{R}^{n}$. The sets $B+g, g \in \mathbb{Z}^{n}$ cover all the space $\mathbb{R}^{n}$. For every finite word $v=x_{m} x_{m-1} \ldots x_{1} \in \mathrm{X}^{m}$ and $g \in \operatorname{Dom} \phi^{m}$ denote

$$
\begin{gathered}
B(v, g):=A^{m}\left(B+r_{x_{m}}+A^{-1}\left(r_{x_{m-1}}\right)+\cdots+\phi^{-m+1}\left(r_{x_{1}}\right)+A^{-m}(g)\right)= \\
A^{m}(B)+A^{m}\left(r_{x_{m}}\right)+A^{m-1}\left(r_{x_{-m+1}}\right)+\cdots+A\left(r_{x_{-1}}\right)+g .
\end{gathered}
$$

Since the linear map $A$ is a contraction, the diameter of the set $B(v, g)$ tends to zero as $m \rightarrow+\infty$.

The sets $\{B(v, g)\}_{|v|=m}$ form a covering of the space $\mathbb{R}^{n}$, which is the image of the covering $\left\{B+g: g \in \mathbb{Z}^{n}\right\}$ under $A^{m}$.

Therefore for every vector $z \in \mathbb{R}^{n}$ and every $m \in \mathbb{N}$ there exists a word $v_{m} \in \mathrm{X}^{m}$ and an element $g_{m} \in \operatorname{Dom} \phi^{m}$ such that $z \in B\left(v_{m}, g_{m}\right)$. We have a uniform estimate on the possible values of the difference $z-g_{m}$. Hence, the set of possible values of $g_{m}$ is finite. Therefore we can find a sequence $m_{k}$ such that the sequence $v_{m_{k}}$ is convergent to an element $v \in \mathrm{X}^{-\omega}$ and $g_{m_{k}}=g$ is constant. Now exponential decreasing of the diameter of the set $B\left(v_{m}, g_{m}\right)$ implies that $\Phi(v+g)=z$.

Thus the map $\Phi$ is a continuous bijection from $X_{\mathbb{Z}^{n}}$ to $\mathbb{R}^{n}$. Both spaces are locally compact Hausdorff, therefore it is a homeomorphism. The map $\Phi$ conjugates the action of $\mathbb{Z}^{n}$ on $X_{\mathbb{Z}^{n}}$ to the natural action on $\mathbb{R}^{n}$.

The second statement of the theorem is a direct corollary of the first and definition of $\Phi$.

\section{References}

[Ale83] S. V. Aleshin, A free group of finite automata, Moscow University Mathematics Bulletin 38 (1983), 10-13.

[Ban91] Christoph Bandt, Self-similar sets. V: Integer matrices and fractal tilings of $\mathbb{R}^{n}$, Proc. Am. Math. Soc. 112 (1991), no. 2, 549-562.

[BG00] Laurent Bartholdi and Rostislav I. Grigorchuk, On the spectrum of Hecke type operators related to some fractal groups, Proceedings of the Steklov Institute of Mathematics 231 (2000), 5-45.

[BG02] $\quad$ On parabolic subgroups and Hecke algebras of some fractal groups, Serdica Math. J. 28 (2002), 47-90.

[BGŠ03] Laurent Bartholdi, Rostislav I. Grigorchuk, and Zoran Šuniḱ, Branch groups, Handbook of Algebra, Vol. 3, North-Holland, Amsterdam, 2003, pp. 989-1112.

[BJ99] Ola Bratteli and Palle E. T. Jorgensen, Iterated function systems and permutation representations of the Cuntz algebra, vol. 139, Memoirs of the American Mathematical Society, no. 663, A. M. S., Providence, Rhode Island, 1999.

[BN03] Evgen Bondarenko and Volodymyr Nekrashevych, Post-critically finite selfsimilar groups, Algebra and Discrete Mathematics 2 (2003), no. 4, 21-32.

[Bou71] Nicolas Bourbaki, Éléments de mathématique. Topologie générale. Chapitres 1 à 4, Hermann, Paris, 1971. 
[BS98] Andrew M. Brunner and Said N. Sidki, The generation of $G L(n, Z)$ by finite state automata, Internat. J. Algebra Comput. 8 (1998), no. 1, 127-139.

[BV05] Laurent Bartholdi and Bálint Virág, Amenability via random walks, Duke Math. J. 130 (2005), no. 1, 39-56.

[Eil74] Samuel Eilenberg, Automata, languages and machines, vol. A, Academic Press, New York, London, 1974.

[Eng77] Ryszard Engelking, General topology, Monografie Matematyczne, vol. 60, Państwowe Wydawnictwo Naukove, Warszawa, 1977.

[Fat20] Pierre Fatou, Sur les équations fonctionnelles, Bull. Soc. Math. France 48 (1920), 33-94.

[FG91] Jacek Fabrykowski and Narain D. Gupta, On groups with sub-exponential growth functions. II, J. Indian Math. Soc. (N.S.) 56 (1991), no. 1-4, 217228 .

[For81] Otto Forster, Lectures on Riemann surfaces, Graduate Texts in Mathematics, vol. 81, New York - Heidelberg - Berlin: Springer-Verlag, 1981.

[GLSŻ00] Rostislav I. Grigorchuk, Peter Linnell, Thomas Schick, and Andrzej Żuk, On a question of Atiyah, C. R. Acad. Sci. Paris Sér. I Math. 331 (2000), no. 9, 663-668.

[GM05] Yair Glasner and Shahar Mozes, Automata and square complexes, Geom. Dedicata 111 (2005), 43-64.

[GNS00] Rostislav I. Grigorchuk, Volodymyr V. Nekrashevich, and Vitaliu I. Sushchanskii, Automata, dynamical systems and groups, Proceedings of the Steklov Institute of Mathematics 231 (2000), 128-203.

[Gri80] Rostislav I. Grigorchuk, On Burnside's problem on periodic groups, Functional Anal. Appl. 14 (1980), no. 1, 41-43.

[Gri88] Semigroups with cancellations of polynomial growth, Mat. Zametki 43 (1988), no. 3, 305-319, 428.

[GS83] Narain D. Gupta and Said N. Sidki, On the Burnside problem for periodic groups, Math. Z. 182 (1983), 385-388.

[Guc70] J. Guckenheimer, Endomorphisms of the Riemann sphere, Global Analysis, Proc. Sympos. Pure Math., vol. 14, American Math. Soc., Providence, Rhode Island, 1970, pp. 95-123.

[GŻ01] Rostislav I. Grigorchuk and Andrzej Żuk, The lamplighter group as a group generated by a 2 -state automaton and its spectrum, Geom. Dedicata $\mathbf{8 7}$ (2001), no. 1-3, 209-244.

[Ġ̇02a] On a torsion-free weakly branch group defined by a three state automaton, Internat. J. Algebra Comput. 12 (2002), no. 1, 223-246.

[GŻ02b] Rostislav I. Grigorchuk. and Andrzej Żuk, Spectral properties of a torsionfree weakly branch group defined by a three state automaton, Computational and Statistical Group Theory (Las Vegas, NV/Hoboken, NJ, 2001), Contemp. Math., vol. 298, Amer. Math. Soc., Providence, RI, 2002, pp. 5782 .

[Har00] Pierre de la Harpe, Topics in geometric group theory, University of Chicago Press, 2000.

[Knu69] Donald E. Knuth, The art of computer programming, Vol. 2, Seminumerical algorithms, Addison-Wesley Publishing Company, 1969.

[Kur61] Kazimierz Kuratowski, Topologie, vol. II, Warszawa, 1961.

[LPS88] Alexander Lubotzky, Ralph Philips, and Peter Sarnak, Ramanujan graphs, Combinatorica 8 (1988), no. 3, 261-277. 
[Lyu87] M.Yu. Lyubich, The dynamics of rational transforms: the topological picture, Russ. Math. Surv. 41 (1987), no. 4, 43-117.

[Mil99] John W. Milnor, Dynamics in one complex variable. Introductory lectures, Wiesbaden: Vieweg, 1999.

[Nek02] Volodymyr V. Nekrashevych, Iterated monodromy groups, preprint, Geneva University, available at http://www . unige.ch/math/biblio/preprint/2002/img.ps, 2002.

[Nek05] Volodymyr Nekrashevych, Self-similar groups, Mathematical Surveys and Monographs, vol. 117, Amer. Math. Soc., Providence, RI, 2005.

[NS04] Volodymyr Nekrashevych and Said Sidki, Automorphisms of the binary tree: state-closed subgroups and dynamics of 1/2-endomorphisms, Groups: Topological, Combinatorial and Arithmetic Aspects (T. W. Müller, ed.), LMS Lecture Notes Series, vol. 311, 2004, pp. 375-404.

[Poi93] Alfredo Poirier, On Post Critically Finite Polynomials. Part One: Critical Portraits, arXiv:math.DS/9305207 v1, 1993.

[Sid87a] Said N. Sidki, On a 2-generated infinite 3-group: subgroups and automorphisms, J. Algebra 110 (1987), no. 1, 24-55.

[Sid87b] On a 2-generated infinite 3-group: the presentation problem, $J$. Algebra 110 (1987), no. 1, 13-23.

[Sid98] Regular trees and their automorphisms, Monografias de Matematica, vol. 56, IMPA, Rio de Janeiro, 1998.

[Sid00] $\quad$ Automorphisms of one-rooted trees: growth, circuit structure and acyclicity, J. of Mathematical Sciences (New York) 100 (2000), no. 1, 19251943.

[Sus98] Vitaliı̆ I. Sushchansky, Groups of automatic permutations, Dop. NAN Ukrainy (1998), no. 6, 47-51, (in Ukrainian).

[Vin95] Andrew Vince, Rep-tiling Euclidean space, Aequationes Mathematicae 50 (1995), 191-213.

[Vin00] Digit tiling of Euclidean space, Directions in Mathematical Quasicrystals, Amer. Math. Soc., Providence, RI, 2000, pp. 329-370.

[VV06] M. Vorobets and Y. Vorobets, On a free group of transformations defined by an automaton, Preprint, (arXiv:math.GR/0601231), 2006.

[Yac73] M. V. Yacobson, On the question of topological classification of rational mappings of the Riemann sphere, Uspekhi Mat. Nauk 28 (1973), no. 2, 247-248.

[Yac80] Markov partitions for rational endomorphisms of the Riemann sphere, Multicomponent Random Systems, Dekker, New York, 1980, pp. 381-396. 